\title{
A common formalism for the integral formulations of the forward EEG problem
}

\author{
Jan Kybic ${ }^{\dagger *}$, Maureen Clerc $^{\dagger}$, Toufic Abboud, Olivier Faugeras, Renaud Keriven, Théo Papadopoulo
}

\begin{abstract}
The forward electro-encephalography (EEG) problem involves finding a potential $V$ from the Poisson equation $\nabla \cdot(\sigma \nabla V)=f$, in which $f$ represents electrical sources in the brain, and $\sigma$ the conductivity of the head tissues. In the piecewise constant conductivity head model, this can be accomplished by the Boundary Element Method (BEM) using a suitable integral formulation. Most previous work uses the same integral formulation, corresponding to a double-layer potential. In this article we present a conceptual framework based on a well-known theorem (Theorem 1) that characterizes harmonic functions defined on the complement of a bounded smooth surface. This theorem says that such harmonic functions are completely defined by their values and those of their normal derivatives on this surface. It allows us to cast the previous BEM approaches in a unified setting and to develop two new approaches corresponding to different ways of exploiting the same theorem. Specifically, we first present a dual approach which involves a single-layer potential. Then, we propose a symmetric formulation, which combines single and double-layer potentials, and which is new to the field of EEG, although it has been applied to other problems in electromagnetism. The three methods have been evaluated numerically using a spherical geometry with known analytical solution, and the symmetric formulation achieves a significantly higher accuracy than the alternative methods. Additionally, we present results with realistically shaped meshes. Beside providing a better understanding of the foundations of BEM methods, our approach appears to lead also to more efficient algorithms.
\end{abstract}

Index Terms-Boundary Element Method, Poisson equation, integral method, EEG

\section{INTRODUCTION}

Electroencephalography (EEG) [1] is a non-invasive method of measuring the electrical activity of the brain. To reconstruct the sources in the brain (the inverse problem), an accurate forward model of the head must be established first. The so called forward problem addresses the calculation of the electric potential $V$ on the scalp for a known configuration of the sources, provided that the physical properties of the head tissues (conductivities) are known. Note that the same forward model can be used for magnetoencephalography (MEG) [2,3], since the magnetic field $\mathbf{B}$ can be calculated from the potential $V$ by simple integration [4].

\section{A. Problem definition}

The quasi-static approximation of Maxwell equations [2,5] in a conducting environment yields the fundamental Poisson

${ }^{\dagger}$ corresponding authors. Odyssée Laboratory - ENPC/ENS/INRIA. Address: INRIA, 2004 Route des Lucioles, BP93, 06902 Sophia-Antipolis, France. email: Maureen.Clerc@ sophia.inria.fr, phone: +33-492 3877 35. fax: +33-492 3878 45. * currently Center for Applied Cybernetics, Faculty of Electrical Engineering, Czech Technical University in Prague, Czech Republic, sponsored by the Czech Ministry of Education under Project LN00B096. email: kybic@fel.cvut.cz, tel: +420224357264.

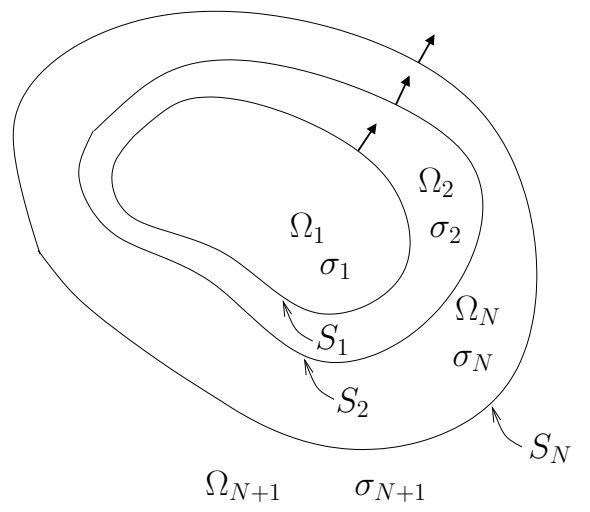

Fig. 1. The head is modeled as a set of nested regions $\Omega_{1}, \ldots, \Omega_{N+1}$ with constant conductivities $\sigma_{1}, \ldots, \sigma_{N+1}$, separated by interfaces $S_{1}, \ldots, S_{N}$. Arrows indicate the normal directions (outward).

equation

$$
\nabla \cdot(\sigma \nabla V)=f=\nabla \cdot \mathbf{J}^{\mathrm{p}} \quad \text { in } \mathbb{R}^{3}
$$

where $\sigma\left[(\Omega \cdot \mathrm{m})^{-1}\right]$ is the conductivity and $f$ is the divergence of the current source density $\mathbf{J}^{\mathrm{p}}\left[\mathrm{A} / \mathrm{m}^{2}\right]$, both supposed known in the forward problem; $V$ (in Volts) is the unknown electric potential.

We shall concentrate on a head model with piecewiseconstant conductivity, such as shown in Fig. 1, with connected open sets $\Omega_{i}$, separated by surfaces $S_{j}$. Note that for the sake of notational simplicity, in this article we only consider nested regions with interfaces $S_{i}=\partial \Omega_{i} \cap \partial \Omega_{i+1}$. However, extension to other topologies is possible and straightforward.

The outermost volume $\Omega_{N+1}$ extends to infinity and in the EEG problem treated here the corresponding conductivity $\sigma_{N+1}$ (the conductivity of air) is considered to be 0 . This implies that there can be no source in $\Omega_{N+1}$. We also assume that there are no charges there. The extension to $\sigma_{N+1} \neq 0$ is trivial.

\section{B. Notation}

We use the notation $\partial_{\mathbf{n}} V=\mathbf{n} \cdot \nabla V$ to denote the partial derivative of $V$ in the direction of a unit vector $\mathbf{n}$, normal to an interface $S_{j}, j=1, \ldots, N$. A function $f$ considered on the interface $S_{j}$ will be denoted $f_{S_{j}}$. We define the jump of a function $f: \mathbb{R}^{3} \rightarrow \mathbb{R}$ at interface $S_{j}$ as

$$
[f]_{j}=f_{S_{j}}^{-}-f_{S_{j}}^{+},
$$


the functions $f^{-}$and $f^{+}$on $S_{j}$ being respectively the interior and exterior limits of $f$ :

$$
\text { for } \mathbf{r} \in S_{j}, \quad f_{S_{j}}^{ \pm}(\mathbf{r})=\lim _{\alpha \rightarrow 0^{ \pm}} f(\mathbf{r}+\alpha \mathbf{n}) .
$$

Note that these quantities depend on the orientation of $\mathbf{n}$, which is taken outward by default, as shown in Fig. 1.

\section{Connected Poisson problems}

Since the conductivity is supposed to be piecewise constant, we can factor out $\sigma$ from (1) to yield a set of Poisson problems coupled by boundary conditions

$$
\begin{array}{rlrl}
\sigma_{i} \Delta V & =f & & \text { in } \Omega_{i}, \text { for all } i=1, \ldots, N \\
\Delta V=0 & & \text { in } \Omega_{N+1} \\
{[V]_{j}=\left[\sigma \partial_{\mathbf{n}} V\right]_{j}=0} & & \text { on } S_{j}, \text { for all } j=1, \ldots, N
\end{array}
$$

The equation (3) is a Laplace equation arising from the fact that the conductivity is assumed to be zero and no charges present outside the head. Physically, the boundary condition $[V]_{j}=0$ imposes the continuity of the potential across the interfaces. The quasi-static assumption implies the continuity of the current (charge) flow across the interfaces, which is expressed by the second boundary condition $\left[\sigma \partial_{\mathbf{n}} V\right]_{j}=0$, as $\sigma \partial_{\mathbf{n}} V=\mathbf{n} \cdot \sigma E$ is precisely the density of current. Mathematically, both boundary conditions come from considering (1) on the boundaries.

\section{Boundary Element Method}

The Boundary Element Method (BEM) [6,7] is today a classical way of solving the forward problem. The advantage of the BEM with respect to the finite difference method (FDM) or the finite element method (FEM) resides in the fact that it only uses as unknowns the values on the interfaces between regions with different conductivities, as opposed to considering values everywhere in the volume. This reduces the dimensionality of the problem and the number of unknowns, and only requires the use of surface triangulation meshes, avoiding the difficult construction of the volume discretization needed for the FEM.

\section{E. Inaccuracy of BEM implementations}

So far the main disadvantage of using BEM in the EEG forward problem has been that in all known implementations the precision drops unacceptably when the distance $d$ of the source to one of the surfaces becomes comparable to the size $h$ of the triangles in the mesh (see also Section V-B.1). This seriously hinders the usefulness of the BEM, as the sources which are measured by EEG are often supposed to lie in the cortex, which is only a few millimeters thick. Although the problem is widely acknowledged [8-11], no satisfactory solution has been found so far. Replacing the collocation by the Galerkin method $[8,12]$ for the resolution of the integral equations improves the precision only partially. The problem has largely been disregarded, or sometimes avoided at the expense of excessively simplifying the model: some authors propose to omit either the outer cortex boundary, or the skull, claiming that these simplifications are inconsequential for the localization accuracy $[13,14]$. Unfortunately, our experiments do not support this claim and there is direct and indirect evidence $[15,16]$ to show that accurate models are essential for accurate reconstruction. Note, however, that the MEG reconstruction is reportedly less affected by modeling errors than the EEG.

\section{F. Proposed new integral formulation}

As far as we know, all variants of the BEM applied to the EEG forward problem are based on the same integral formulation, introduced by Geselowitz [17] in 1967. However, this integral formulation is by no means the only one available. We show that the classical formulation corresponds to a doublelayer potential approach. We propose a dual formulation using a single-layer potential. Finally, we present a new formulation, combining single and double-layer potentials. This new approach leads to a symmetric system and turns out to be numerically significantly more accurate than the other two formulations.

\section{G. Existing work}

There is a large body of literature describing BEM implementations using the double-layer potential formulation for forward and inverse EEG problems [8, 12, 18-22].

The symmetric formulation has existed in the BEM community for some time [7,23-25], and the single-layer potential formulation has been used for solving elasticity problems $[6$, 7]. However, to the best of our knowledge, neither the symmetric approach nor the single-layer formulation have so far been applied to the EEG problem.

\section{H. Organization of this article}

We start in Section II by presenting the mathematical results needed for the Boundary Element Method. Section III presents the classical double-layer potential formulation together with its dual formulation in terms of a single-layer potential, and the new symmetric integral formulation, which combines single and double-layer potentials. The discretization and implementation are described in Section IV, followed by experimental results in Section V. Technical justifications and remarks relative to Section II are detailed in Appendix A and can be skipped at first reading.

\section{REPRESENTATION THEOREM}

The power of the Boundary Element Method is in its conciseness, since it only requires to solve for values defined on surfaces instead of values defined in the volume. The key to this dimension reduction resides in a fundamental representation theorem $[6,7]$, which we recall in this section.

We define a Green function

$$
G(\mathbf{r})=\frac{1}{4 \pi\|\mathbf{r}\|} \quad \text { satisfying } \quad-\Delta G=\delta_{0} .
$$


Given a regular boundary (surface) $\partial \Omega$, we introduce four integral operators $\mathcal{D}, \mathcal{S}, \mathcal{N}, \mathcal{D}^{*}$, which map a scalar function $f$ on $\partial \Omega$ to another scalar function on $\partial \Omega$ :

$$
\begin{gathered}
(\mathcal{D} f)(\mathbf{r})=\int_{\partial \Omega} \partial_{\mathbf{n}^{\prime}} G\left(\mathbf{r}-\mathbf{r}^{\prime}\right) f\left(\mathbf{r}^{\prime}\right) \mathrm{d} s\left(\mathbf{r}^{\prime}\right), \\
(\mathcal{S} f)(\mathbf{r})=\int_{\partial \Omega} G\left(\mathbf{r}-\mathbf{r}^{\prime}\right) f\left(\mathbf{r}^{\prime}\right) \mathrm{d} s\left(\mathbf{r}^{\prime}\right), \\
(\mathcal{N} f)(\mathbf{r})=\int_{\partial \Omega} \partial_{\mathbf{n}, \mathbf{n}^{\prime}} G\left(\mathbf{r}-\mathbf{r}^{\prime}\right) f\left(\mathbf{r}^{\prime}\right) \mathrm{d} s\left(\mathbf{r}^{\prime}\right), \\
\left(\mathcal{D}^{*} f\right)(\mathbf{r})=\int_{\partial \Omega} \partial_{\mathbf{n}} G\left(\mathbf{r}-\mathbf{r}^{\prime}\right) f\left(\mathbf{r}^{\prime}\right) \mathrm{d} s\left(\mathbf{r}^{\prime}\right) .
\end{gathered}
$$

where $\mathbf{n}$, resp. $\mathbf{n}^{\prime}$, is the outward normal vector at position $\mathbf{r}$, resp. $\mathbf{r}^{\prime}$. Note that the operator $\mathcal{D}^{*}$ is the transpose (adjoint) of $\mathcal{D}$ with respect to the $L^{2}(\partial \Omega)$ scalar product $\langle f, g\rangle=$ $\int_{\partial \Omega} f(\mathbf{r}) g(\mathbf{r}) \mathrm{d} s\left(\mathbf{r}^{\prime}\right)$. With a slight abuse of notation, we will also consider the values of the above-defined $(\mathcal{D} f)(\mathbf{r})$ and $(\mathcal{S} f)(\mathbf{r})$ at any point in $\mathbb{R}^{3}$, not necessarily on the boundary $\partial \Omega$. The same generalization can also be applied to $\left(\mathcal{D}^{*} f\right)(\mathbf{r})$, and to $(\mathcal{N} f)(\mathbf{r})$, choosing an arbitrary smooth vector field $\mathbf{n}(\mathbf{r})$.

To simplify the treatment and avoid ambiguity, we choose to work with potential functions vanishing at infinity; more precisely, we say that a function $u$ satisfies condition $\mathscr{H}$, if simultaneously

$$
\left\{\begin{array}{l}
\lim _{r \rightarrow \infty} r|u(\mathbf{r})|<\infty \\
\lim _{r \rightarrow \infty} r \frac{\partial u}{\partial r}(\mathbf{r})=0
\end{array},\right.
$$

where $r=\|\mathbf{r}\|$, and $\frac{\partial u}{\partial r}(\mathbf{r})$ denotes the partial derivative of $u$ in the radial direction. The Green function $G$ in (5) satisfies $\mathscr{H}$. The condition $\mathscr{H}$ corresponds to the physical intuition that a static field far away from all charges is zero. This goes together with the hypothesis we need in order to make our initial physical problem uniquely solvable, namely that we are only interested in the field due to sources inside our bounded volumes, i.e. inside the head.

We are now ready to state the fundamental representation theorem on which the Boundary Element Method is based.

Theorem 1 (Representation Theorem) Let $\Omega \subseteq \mathbb{R}^{3}$ be a bounded open set with a regular boundary $\partial \Omega$. Let $u$ : $\left(\mathbb{R}^{3} \backslash \partial \Omega\right) \rightarrow \mathbb{R}$ be a harmonic function $\left(\Delta u=0\right.$ in $\left.\mathbb{R}^{3} \backslash \partial \Omega\right)$, satisfying the $\mathscr{H}$ condition, and let further $p(\mathbf{r}) \stackrel{\text { def }}{=} \partial_{\mathbf{n}} u(\mathbf{r})$. Then

$$
\begin{aligned}
& -p=\quad+\mathcal{N}[u] \quad-\mathcal{D}^{*}[p] \quad \text { for } \mathbf{r} \notin \partial \Omega \\
& u=\quad-\mathcal{D}[u] \quad+\mathcal{S}[p] \\
& -p^{ \pm}=\quad+\mathcal{N}[u] \quad+\left( \pm \frac{\mathcal{J}}{2}-\mathcal{D}^{*}\right)[p] \quad \text { for } \mathbf{r} \in \partial \Omega \\
& u^{ \pm}=\left(\mp \frac{\mathcal{J}}{2}-\mathcal{D}\right)[u] \quad+\mathcal{S}[p]
\end{aligned}
$$

where J denotes the identity operator.

The Theorem holds in particular for the hollow ball topology depicted in Figure 2, i.e. for disjoint open sets $\Omega_{1}, \Omega_{2}, \Omega_{3}$

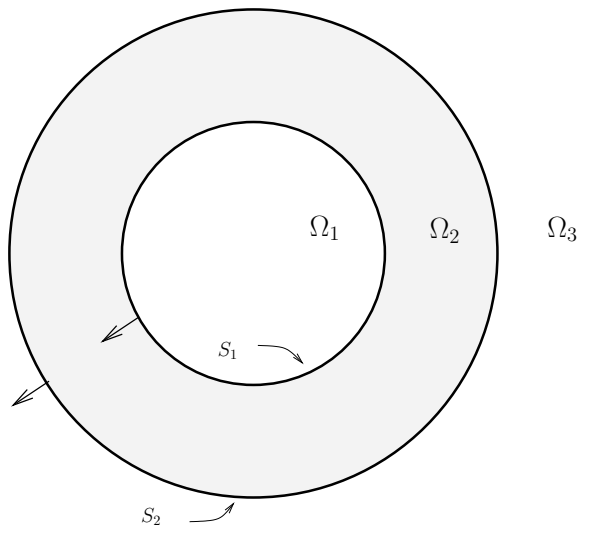

Fig. 2. Two-dimensional slice through a volume $\Omega_{2}$ (delimited by surfaces $S_{1}, S_{2}$ ) with a hollow ball topology. Arrows denote the normal orientation.

such that $\bar{\Omega}_{1} \cup \bar{\Omega}_{2} \cup \Omega_{3}=\mathbb{R}^{3}$, separated by regular boundaries $\partial \Omega_{1} \cap \partial \Omega_{2}=S_{1}, \partial \Omega_{2} \cap \partial \Omega_{3}=S_{2}$, and $\partial \Omega_{1} \cap \partial \Omega_{3}=\emptyset$, if we set $\partial \Omega=S_{1} \cup S_{2}$. This result will be used in Section III-G to establish the symmetric formulation.

Theorem 1 shows that any harmonic function $u$ in $\mathbb{R}^{3} \backslash \partial \Omega$ satisfying $\mathscr{H}$ is determined everywhere by its jump and the jump of its derivative across the boundary $\partial \Omega$, whether $\partial \Omega$ is a single surface, or two surfaces as in the case of Fig. 2. This is a very deep result, showing the strong constraints imposed by the harmonicity. It helps us to understand why we can solve a $3 \mathrm{D}$ problem by only considering quantities on a $2 \mathrm{D}$ surface. For additional notes and a sketch of a proof, we refer the reader to Appendix A.

\section{A. Single and double-layer potentials}

From the equations (7) in Theorem 1, we see that the harmonic function $u$ can be represented using two functions $\mu=-[u]$ and $\xi=[p]$ defined on $\partial \Omega$. Historically, the $\delta \xi$ part of (7) is called a single-layer potential. The single-layer potential is continuous when crossing $\partial \Omega$, while its normal derivative is not; $[\delta \xi]_{\partial \Omega}=0,\left[\partial_{\mathbf{n}} \delta \xi\right]_{\partial \Omega}=\xi$. On the other hand, the second part, $\mathcal{D} \mu$, which is called a double-layer potential, jumps over $\partial \Omega$, while its normal derivative does not; $[\mathcal{D} \mu]_{\partial \Omega}=-\mu,\left[\partial_{\mathbf{n}} \mathcal{D} \mu\right]_{\partial \Omega}=0$. More detail on single and double-layer potentials is provided in Appendix A.

To apply the single/double-layer potentials to our nestedregion model in Fig. 1, we simply add up the contributions from all interfaces, $u_{s}=\sum_{i} \delta \xi_{S_{i}}$ resp. $u_{d}=\sum_{i} \mathcal{D} \mu_{S_{i}}$. This yields single, resp. double-layer potentials with the same jump properties as in the single interface case (see Appendix B). In particular, we shall need further on the following two relations, easily obtainable from (7) by additivity:

$$
\begin{aligned}
& \partial_{\mathbf{n}} u_{s}^{ \pm}(\mathbf{r})=\mp \frac{\xi_{S_{j}}}{2}+\sum_{i=1}^{N} \mathcal{D}_{j i}^{*} \xi_{S_{i}} \quad \text { for } \mathbf{r} \in S_{j} \\
& u_{d}^{ \pm}(\mathbf{r})= \pm \frac{\mu_{S_{j}}}{2}+\sum_{i=1}^{N} \mathcal{D}_{j i} \mu_{S_{i}} \quad \text { for } \mathbf{r} \in S_{j} .
\end{aligned}
$$

The operators $\mathcal{D}_{j i}^{*}$ and $\mathcal{D}_{j i}$ are restrictions of $\mathcal{D}^{*}$ and $\mathcal{D}$ : they act on a function defined on $S_{i}$ and yield a function defined 
on $S_{j}$. This convention is used consistently in the rest of this paper.

\section{INTEGRAL FORMULATIONS}

Let us use Theorem 1 to obtain integral formulations for the original multiple interface problem (4). We now need to cope with the presence of sources, which make the solution non-harmonic. Our starting point is a homogeneous solution $v$, which takes the source terms into account, but does not necessarily respect all boundary conditions. Then we add to $v$ a harmonic function $u$ to obtain a complete solution $V$ which simultaneously respects the Poisson equation $\sigma_{i} \Delta V=f$ in all $\Omega_{i}$, the boundary conditions (4), and the equation (3). Three different ways of achieving this are described in this section. We shall always assume that $V$ satisfies condition $\mathscr{H}$, which amounts to imposing a zero potential infinitely far from all sources.

\section{A. Dipolar and surface sources}

The source model most commonly used to represent electrical activity in the brain is a "current dipole"1 [3]. It represents an infinitely small oriented source of current positioned at $\mathbf{r}_{0}$, with dipolar moment $\mathbf{q}$, and is defined by $\mathbf{J}_{\text {dip }}(\mathbf{r})=\mathbf{q} \delta_{\mathbf{r}_{0}}(\mathbf{r})$. The corresponding source term in the Poisson equation is $f_{\text {dip }}=\nabla \cdot \mathbf{J}_{\text {dip }}=\mathbf{q} \cdot \nabla \delta_{\mathbf{r}_{0}}$, which yields the homogeneous domain potential

$$
v_{\text {dip }}(\mathbf{r})=\frac{1}{4 \pi} \frac{\mathbf{q} \cdot\left(\mathbf{r}-\mathbf{r}_{0}\right)}{\left\|\mathbf{r}-\mathbf{r}_{0}\right\|^{3}} .
$$

The dipolar source is physiologically plausible in that it represents movement of charges, not their creation. At sufficiently long time scale it approximates the neuronal pulse trains.

Sources on cortex surface and perpendicular to it can be also modeled as $\mathbf{J}_{\text {surf }}(\mathbf{r})=j(\mathbf{r}) \mathbf{n}_{P}(\mathbf{r}) \delta_{P}(\mathbf{r})$ with scalar surface current density $j$ on a patch $P$. The corresponding homogeneous potential $v_{\text {surf }}$ is then calculated by integration over $P$ :

$$
v_{\text {surf }}(\mathbf{r})=\frac{1}{4 \pi} \int_{\mathbf{r}^{\prime} \in P} \frac{\mathbf{n}_{P}\left(\mathbf{r}^{\prime}\right) \cdot\left(\mathbf{r}-\mathbf{r}^{\prime}\right)}{\left\|\mathbf{r}-\mathbf{r}^{\prime}\right\|^{3}} j\left(\mathbf{r}^{\prime}\right) \mathrm{d} \mathbf{r}^{\prime}
$$

Finally, we can consider a completely general volume current density $\mathbf{J}^{p}$, yielding a source term $f=\nabla \cdot \mathbf{J}^{p}$ and a potential $v=-f * G$. (See also next Section.)

\section{B. Homogeneous solution}

We decompose the source $f$ from (1) into $f=\sum_{i=1}^{N} f_{\Omega_{i}}$ such that $f_{\Omega_{i}}=f \cdot 1_{\Omega_{i}}$, where $1_{\Omega_{i}}$ is the indicator function of $\Omega_{i}$ (hence $f_{\Omega_{i}}=0$ outside $\Omega_{i}$ ), $i=1, \cdots, N$. Recall that no source lies in $\Omega_{N+1}$; we also assume that no source lies on any boundary $S_{i}$.

For each partial source term $f_{\Omega_{i}}$ we calculate the homogeneous medium solution $v_{\Omega_{i}}(\mathbf{r})=-f_{\Omega_{i}} * G(\mathbf{r})$. The convolution theorem and the properties of the Green function (5) show that $\Delta v_{\Omega_{i}}=-f_{\Omega_{i}} * \Delta G=f_{\Omega_{i}}$. They are harmonic in

${ }^{1}$ This is a traditional name, used because the quantity $\mathbf{q}$ has the units of $[\mathrm{A} \cdot \mathrm{m}]$.
$\mathbb{R} \backslash \bar{\Omega}_{i}, i=1, \cdots, N$ and hence also in $\Omega_{N+1}$. Thanks to the choice of $G$ in (5), the functions $v_{\Omega_{i}}$ satisfy condition $\mathscr{H}$, provided that the $f_{\Omega_{i}}$ are compactly supported. This is true by construction for $\Omega_{1}, \ldots, \Omega_{N}$ since each of these domains is bounded.

\section{Multiple domains}

There are various ways of combining the individual homogeneous solutions $v_{\Omega_{i}}$ from domains $\Omega_{i}$ into a global homogeneous $v$. First we consider a function $v_{s}$ constructed as:

$$
v_{s}=\sum_{i=1}^{N} v_{\Omega_{i}} / \sigma_{i}
$$

We easily verify that it satisfies the Poisson equation $\sigma \Delta v_{s}=$ $f$ in each $\Omega_{i}, i=1, \ldots, N$ :

$$
\sigma \Delta v_{s}=\sigma \sum_{i=1}^{N} \Delta v_{\Omega_{i}} / \sigma_{i}=\sigma \sum_{i=1}^{N} \frac{f_{\Omega_{i}}}{\sigma_{i}}=\sum_{i=1}^{N} f_{\Omega_{i}}=f
$$

in each $\Omega_{i}, i=1, \ldots, N$. According to the previous section, all functions $v_{\Omega_{i}}, i=1, \ldots, N$ are harmonic in $\Omega_{N+1}$, hence so is $v_{s}$.

The function $v_{s}$ and its derivative $\partial_{\mathbf{n}} v_{s}$ are continuous across each $S_{j}$. In other words, $v_{s}$ satisfies the boundary conditions $\left[v_{s}\right]_{j}=0$ and $\left[\partial_{\mathbf{n}} v_{s}\right]_{j}=0$ for all $j$, but not the boundary condition $\left[\sigma \partial_{\mathbf{n}} v_{s}\right]_{j}=0$. The function $v_{s}$ will be used in the single-layer approach, Section III-D, whence the subscript $s$.

In a dual fashion, we would like to consider the function $\tilde{v}_{d}(\mathbf{r})=\sigma^{-1}(\mathbf{r}) \sum_{i=1}^{N} v_{\Omega_{i}}$ that satisfies $\sigma \Delta \tilde{v}_{d}=f$ and the boundary condition $\left[\sigma \partial_{\mathbf{n}} \tilde{v}_{d}\right]_{j}=0$. Unfortunately, $\tilde{v}_{d}$ is not properly defined in $\Omega_{N+1}$ where $\sigma=0$. Instead, we introduce a function

$$
v_{d}=\sum_{i=1}^{N} v_{\Omega_{i}}
$$

that satisfies the Poisson equation $\Delta v_{d}=f$ and the boundary conditions $\left[v_{d}\right]_{j}=0$ and $\left[\partial_{\mathbf{n}} v_{d}\right]_{j}=0$ on each surface $S_{j} \cdot v_{d}$ is harmonic in $\Omega_{N+1}$ for the same reasons as $v_{s}$. This function is used in the double-layer approach, Section III-E.

\section{Single-layer approach}

A natural approach for solving (1) consists in representing the potential $V$ in a way which automatically satisfies $[V]_{j}=0$ and then adjusting the harmonic part so that the remaining boundary conditions, $\left[\sigma \partial_{\mathbf{n}} V\right]_{j}=0$, are satisfied as well. We consider $u_{s}=V-v_{s}$, with $v_{s}$ defined in (12). By construction, $u_{s}$ is harmonic in $\Omega=\Omega_{1} \cup \ldots \cup \Omega_{N}$, since in each $\Omega_{i}$ we have $\sigma_{i} \Delta u_{s}=\sigma_{i} \Delta V-\sigma_{i} \Delta v_{s}=f_{\Omega_{i}}-f_{\Omega_{i}}=0$. It is also harmonic in $\Omega_{N+1}$, as both $V$ and $v_{s}$ are harmonic there. Since $[V]_{j}=0$ and $\left[v_{s}\right]_{j}=0$ (Section III-C), we conclude that $\left[u_{s}\right]_{j}=0$ across all surfaces $S_{j}$. This means that $u_{s}$ is a single-layer potential for $\Omega=\Omega_{1} \cup \ldots \cup \Omega_{N+1}$ with the corresponding boundary $\partial \Omega=S_{1} \cup \ldots \cup S_{N}$ ( cf Section IIA). 
We then use the second set of boundary conditions, $\left[\sigma \partial_{\mathbf{n}} V\right]=0$, implying that $\left[\sigma \partial_{\mathbf{n}} u_{s}\right]=-\left[\sigma \partial_{\mathbf{n}} v_{s}\right]$. We express $\left[\sigma \partial_{\mathbf{n}} u_{s}\right]$ as a function of known quantities:

$$
\left[\sigma \partial_{\mathbf{n}} u_{s}\right]_{j}=-\left[\sigma \partial_{\mathbf{n}} v_{s}\right]_{j}=-\left(\sigma_{j}-\sigma_{j+1}\right) \partial_{\mathbf{n}} v_{s} \quad \text { on } S_{j}
$$

since $\partial_{\mathbf{n}} v_{s}$ does not "jump" across $S_{j}$ (Section III-C). Equation (8) provides the normal derivative of the single-layer potential $u_{s}$. Writing $\xi_{S_{j}}=\left[\partial_{\mathbf{n}} u_{s}\right]_{j}$,

$$
\begin{aligned}
{\left[\sigma \partial_{\mathbf{n}} u_{s}\right]_{j}=} & \sigma_{j} \partial_{\mathbf{n}} u_{s}^{-}-\sigma_{j+1} \partial_{\mathbf{n}} u_{s}^{+}= \\
& \frac{\sigma_{j}+\sigma_{j+1}}{2} \xi_{S_{j}}+\left(\sigma_{j}-\sigma_{j+1}\right) \sum_{i=1}^{N} \mathcal{D}_{j i}^{*} \xi_{S_{i}}
\end{aligned}
$$

on all $S_{1}, \ldots, S_{N}$. Combining this result with (14) and dividing by $\left(\sigma_{j}-\sigma_{j+1}\right)$ we obtain ${ }^{2}$

$$
\partial_{\mathbf{n}} v_{s}=\frac{\sigma_{j}+\sigma_{j+1}}{2\left(\sigma_{j+1}-\sigma_{j}\right)} \xi_{S_{j}}-\sum_{i=1}^{N} \mathcal{D}_{j i}^{*} \xi_{S_{i}} \quad \text { on all } S_{j} .
$$

This is a system of $N$ integral equations in the unknown functions $\xi_{S_{j}}$. Its solution is unique up to a constant [7] (see also Appendix C). Once (15) is solved, the potential $u_{s}$ is determined for $\mathbf{r} \in S_{j}$ as

$$
u_{s}(\mathbf{r})=\sum_{i=1}^{N} \mathcal{S}_{j i} \xi_{S_{i}}
$$

and the corresponding values of $V$ follow from $V=v_{s}+u_{s}$.

We observe that $V$ is expressed as an exactly calculable homogeneous medium potential $v_{s}$ plus a correction term $u_{s}$. If the medium is close to homogeneous, the correction is small, which helps to improve the accuracy of this method. This method is to be favored if we are interested in calculating the flow or the current. However, to obtain the potential $V$, an additional computation is necessary.

\section{E. Double-layer approach}

The double-layer approach is dual to the single-layer approach. We use a representation satisfying $\left[\sigma \partial_{\mathbf{n}} V\right]_{j}=0$ by construction and then find conditions on the harmonic part to impose $[V]_{j}=0$ as well. Consider a function $u_{d}=\sigma V-v_{d}$, with $v_{d}$ given by (13). By construction, $u_{d}$ is harmonic in $\Omega=\Omega_{1} \cup \ldots \cup \Omega_{N}$, because in each $\Omega_{i}$ we have $\Delta u_{d}=$ $\sigma_{i} \Delta V-\Delta v_{d}=f_{\Omega_{i}}-f_{\Omega_{i}}=0$. It is also harmonic in $\Omega_{N+1}$, as both $V$ and $v_{d}$ are harmonic there. Since $\left[\sigma \partial_{\mathbf{n}} V\right]_{j}=0$ and $\left[\partial_{\mathbf{n}} v_{d}\right]_{j}=0$ (Section III-C), we conclude that $\left[\partial_{\mathbf{n}} u_{d}\right]_{j}=0$ on all surfaces $S_{j}$. This means that $u_{d}$ is a double-layer potential for $\Omega=\Omega_{1} \cup \ldots \cup \Omega_{N+1}$ with the corresponding boundary $\partial \Omega=S_{1} \cup \ldots \cup S_{N}$. Equation (9) expresses the boundary values of a double-layer representation. We now use the second set of boundary conditions, $[V]_{j}=0$, implying that $\sigma_{j+1}\left(u_{d}+v_{d}\right)^{-}=\sigma_{j}\left(u_{d}+v_{d}\right)^{+}$for all $S_{j}$. (This is equivalent to $\sigma_{j}^{-1}\left(u_{d}+v_{d}\right)^{-}=\sigma_{j+1}^{-1}\left(u_{d}+v_{d}\right)^{+}$for $\sigma \neq 0$ and a natural extension thereof for $\sigma=0$.) We can also express

\footnotetext{
${ }^{2}$ The division by $\left(\sigma_{j}-\sigma_{j+1}\right)$ has been done to simplify the formula. It should not be performed for small values $\left|\sigma_{j}-\sigma_{j+1}\right|$ in order to avoid numerical diffi culties.
}

$\mu_{S_{i}}=-\left[u_{d}\right]=\left(\sigma_{i+1}-\sigma_{i}\right) V_{S_{i}}$, where $V_{S_{i}}$ is the restriction of $V$ to $S_{i}$. This yields

$$
v_{d}=\frac{\sigma_{j}+\sigma_{j+1}}{2} V_{S_{j}}-\sum_{i=1}^{N}\left(\sigma_{i+1}-\sigma_{i}\right) \mathcal{D}_{j i} V_{S_{i}} \quad \text { on each } S_{j} .
$$

The function $v_{d}$ defined in (13) is the solution of $\Delta v_{d}=f$, corresponding to a homogeneous medium with conductivity equal to one. Remembering that $\mathcal{D}_{j i} V_{S_{i}}(\mathbf{r})=\int_{S_{i}} \partial_{\mathbf{n}^{\prime}} G\left(\mathbf{r}-\mathbf{r}^{\prime}\right) V\left(\mathbf{r}^{\prime}\right) d s\left(\mathbf{r}^{\prime}\right)$ for $\mathbf{r} \in S_{j}$, we recognize in (16) the classical integral formulation used for EEG and MEG [2,3,17,19,20]. The advantage of this approach is that it solves directly for $V$ and requires no additional post-processing. As in the single-layer approach, the solution of the system (16) is unique up to a constant [7].

\section{F. Isolated problem approach}

In 1989, Hämäläinen and Sarvas [13] introduced a variation on this double-layer formulation, to improve the precision of the the classical formulation, caused by the low conductivity of the skull compared to the other head tissues. This approach, called Isolated Problem Approach (IPA) or Isolated Skull Approach, is based on the same idea that improves the accuracy of the single-layer method - we express the potential $V$ as a sum of two parts calculated separately with hopefully more precision than calculating the final result directly. In this case, we calculate first the field of the sources considering only the innermost volume and then the appropriate correction.

The IPA is not general in that it assumes the sources to be only in the innermost layer, which is not the case for the more realistic models of the head, where we want to consider sources in the cortex. Also, while it improves the precision in some cases, it reduces it in others [20]. Therefore, we shall not consider IPA in the rest of this article.

\section{G. Symmetric approach}

The symmetric approach, uses both the single and doublelayer potentials. It is based on the classical theory of Newtonian potentials as described in chapter 2 of [26], the work of Nédélec [7] and is also closely related to algorithms in [23, 24]. However, as far as we know, it has so far never been described for the EEG problem. In this approach, we consider in each $\Omega_{1}, \ldots, \Omega_{N}$ the function

$$
u_{\Omega_{i}}= \begin{cases}V-v_{\Omega_{i}} / \sigma_{i} & \text { in } \Omega_{i} \\ -v_{\Omega_{i}} / \sigma_{i} & \text { in } \mathbb{R}^{3} \backslash \bar{\Omega}_{i} .\end{cases}
$$

Each $u_{\Omega_{i}}$ is harmonic in $\mathbb{R}^{3} \backslash \partial \Omega_{i}$. Considering the nested volume model (Fig. 1), the boundary of $\Omega_{i}$ is $\partial \Omega_{i}=S_{i-1} \cup S_{i}$. With respect to the orientations of normals indicated in Fig. 3, the jumps of $u_{\Omega_{i}}$ across $S_{i}$ satisfy the relations

$$
\left[u_{\Omega_{i}}\right]_{i}=V_{S_{i}}, \quad\left[u_{\Omega_{i}}\right]_{i-1}=-V_{S_{i-1}},
$$

and the jumps of their derivatives

$$
\left[\partial_{\mathbf{n}} u_{\Omega_{i}}\right]_{i}=\left(\partial_{\mathbf{n}} V\right)_{S_{i}}^{-}, \quad\left[\partial_{\mathbf{n}} u_{\Omega_{i}}\right]_{i-1}=-\left(\partial_{\mathbf{n}} V\right)_{S_{i-1}}^{+} .
$$




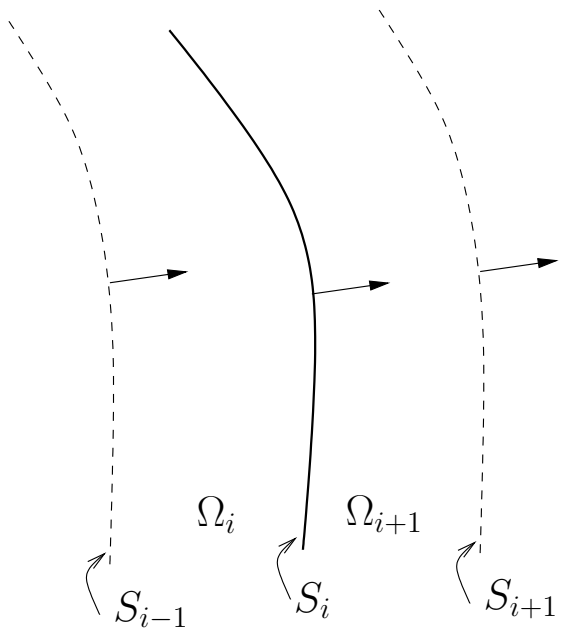

Fig. 3. A detail of the nested volume model. Normal vectors are oriented globally outward, as shown. However, when considering for example the surface $S_{i}$ as the boundary of $\Omega_{i+1}$, the orientation needs to be reversed.

We define $p_{S_{i}}=\sigma_{i}\left[\partial_{\mathbf{n}} u_{\Omega_{i}}\right]_{i}=\sigma_{i}\left(\partial_{\mathbf{n}} V\right)_{S_{i}}^{-}$. Note that since $\left[\sigma \partial_{\mathbf{n}} V\right]=0$ from (4), we have $p_{S_{i}}=\sigma_{i}\left(\partial_{\mathbf{n}} V\right)_{S_{i}}=$ $\sigma_{i+1}\left(\partial_{\mathbf{n}} V\right)_{S_{i}}^{+}$at the interface $S_{i}$. As $u_{\Omega_{i}}$ is harmonic in $\mathbb{R}^{3} \backslash \partial \Omega_{i}$ and satisfies the condition $\mathscr{H}$, we can apply Theorem 1 to obtain the internal limit of $u_{\Omega_{i}}$ on $S_{i}$ :

$$
\left(u_{\Omega_{i}}\right)_{S_{i}}^{-}=\frac{\left[u_{\Omega_{i}}\right]_{\partial \Omega_{i}}}{2}-\mathcal{D}_{\partial \Omega_{i}}\left[u_{\Omega_{i}}\right]_{\partial \Omega_{i}}+\mathcal{S}_{\partial \Omega_{i}}\left[\partial_{\mathbf{n}} u_{\Omega_{i}}\right]_{\partial \Omega_{i}}
$$

If we break down the jump terms across $\partial \Omega_{i}=S_{i-1} \cup S_{i}$ into two parts, corresponding to $S_{i-1}$ and $S_{i}$, and if we take into account identities (17a), we obtain

$$
\begin{array}{r}
\left(u_{\Omega_{i}}\right)_{S_{i}}^{-}=\left(V-v_{\Omega_{i}} / \sigma_{i}\right)_{S_{i}}^{-}=\frac{V_{S_{i}}}{2}+\mathcal{D}_{i, i-1} V_{S_{i-1}}- \\
\mathcal{D}_{i i} V_{S_{i}}-\sigma_{i}^{-1} \mathcal{S}_{i, i-1} p_{S_{i-1}}+\sigma_{i}^{-1} \mathcal{S}_{i i} p_{S_{i}}
\end{array}
$$

A similar analysis applies to $u_{\Omega_{i+1}}$. Theorem 1 gives the external limit of $u_{\Omega_{i+1}}$ on $S_{i}$

$$
\begin{array}{r}
\left(u_{\Omega_{i+1}}\right)_{S_{i}}^{+}=-\frac{\left[u_{\Omega_{i+1}}\right]_{\partial \Omega_{i+1}}}{2}-\mathcal{D}_{\partial \Omega_{i+1}}\left[u_{\Omega_{i+1}}\right]_{\partial \Omega_{i+1}}+ \\
\mathcal{S}_{\partial \Omega_{i+1}}\left[\partial_{\mathbf{n}} u_{\Omega_{i+1}}\right]_{\partial \Omega_{i+1}}
\end{array}
$$

We substitute from (17a) for the values of $\left[u_{\Omega_{i+1}}\right]$ and $\left[\partial_{\mathbf{n}} u_{\Omega_{i+1}}\right]$ and break down the terms on $\partial \Omega_{i+1}=S_{i} \cup S_{i+1}$, to obtain

$$
\begin{aligned}
& \left(u_{\Omega_{i+1}}\right)_{S_{i}}^{+}=\left(V-v_{\Omega_{i+1}} / \sigma_{i+1}\right)_{S_{i}}^{+}= \\
& \frac{V_{S_{i}}}{2}+\mathcal{D}_{i i} V_{S_{i}}-\mathcal{D}_{i, i+1} V_{S_{i+1}}-\sigma_{i+1}^{-1} \mathcal{S}_{i i} p_{S_{i}}+\sigma_{i+1}^{-1} \mathcal{S}_{i, i+1} p_{S_{i+1}}
\end{aligned}
$$

We subtract (18) and (19); given that the functions $V, v_{\Omega_{i+1}}$ and $v_{\Omega_{i}}$ are continuous across $S_{i}$ and their internal and external limits hence coincide, we get

$$
\begin{aligned}
& \sigma_{i+1}^{-1}\left(v_{\Omega_{i+1}}\right)_{S_{i}}-\sigma_{i}^{-1}\left(v_{\Omega_{i}}\right)_{S_{i}}= \\
& \mathcal{D}_{i, i-1} V_{S_{i-1}}-2 \mathcal{D}_{i i} V_{S_{i}}+\mathcal{D}_{i, i+1} V_{S_{i+1}}-\sigma_{i}^{-1} \mathcal{S}_{i, i-1} p_{S_{i-1}} \\
& \quad+\left(\sigma_{i}^{-1}+\sigma_{i+1}^{-1}\right) \mathcal{S}_{i i} p_{S_{i}}-\sigma_{i+1}^{-1} \mathcal{S}_{i, i+1} p_{S_{i+1}}
\end{aligned}
$$

for $i=1, \ldots, N$. Using the same approach, we evaluate the quantities $\left(\sigma_{i} \partial_{\mathbf{n}} u_{\Omega_{i}}\right)_{S_{i}}^{-}=\left(p-\partial_{\mathbf{n}} v_{\Omega_{i}}\right)_{S_{i}}^{-}$and $\left(\sigma_{i+1} \partial_{\mathbf{n}} u_{\Omega_{i+1}}\right)_{S_{i}}^{+}=\left(p-\partial_{\mathbf{n}} v_{\Omega_{i+1}}\right)_{S_{i}}^{+}$using Theorem 1, subtract the resulting expressions and obtain

$$
\begin{aligned}
\left(\partial_{\mathbf{n}} v_{\Omega_{i+1}}\right)_{S_{i}}-\left(\partial_{\mathbf{n}} v_{\Omega_{i}}\right)_{S_{i}}= & \\
\sigma_{i} \mathcal{N}_{i, i-1} V_{S_{i-1}}-\left(\sigma_{i}+\sigma_{i+1}\right) & \mathcal{N}_{i i} V_{S_{i}}+\sigma_{i+1} \mathcal{N}_{i, i+1} V_{S_{i+1}}- \\
\mathcal{D}_{i, i-1}^{*} p_{S_{i-1}} & +2 \mathcal{D}_{i i}^{*} p_{S_{i}}-\mathcal{D}_{i, i+1}^{*} p_{S_{i+1}}
\end{aligned}
$$

for $i=1, \ldots, N$. Here (and in (20)) the terms corresponding to non-existing surfaces $S_{0}, S_{N+1}$ are to be set to zero. Terms involving $p_{S_{N}}$ must also be set to zero, since $\sigma_{N+1}=0$ implies $p_{S_{N}}=0$.

Observe that, unlike in the previous approaches, each surface only interacts with its neighbors, at the cost of considering two sets of unknowns, $V_{S_{i}}$ and $p_{S_{i}}$. Equations (20) and (21) thus lead to a block-diagonal symmetric operator matrix, which is displayed in Fig. 4. Note that the vanishing conductivity $\sigma_{N+1}=0$ is taken into account by effectively chopping off the last line and column of the matrix.

\section{DisCRETIZATION AND IMPLEMENTATION}

The discretization of all the exposed integral methods can be divided into three steps: discretization of the boundaries, discretization of the unknowns, and choice of the test functions, corresponding to the choice of the error measure to discretize the equations.

\section{A. Discretization of the boundaries}

The first step is to approximate the boundaries by surface meshes. Triangulation is used in the vast majority of cases. Higher-order surface elements [22] are rarely used for the EEG problem, despite their potential to improve the modeling accuracy, because of the lack of algorithms to generate curved meshes from the available data (mostly volumes of anatomical MRI [27,28]). As a triangulated surface is not regular, some caution is needed in the application of the continuous equations derived above (cf Appendix D).

\section{B. Discretization of the unknowns}

The second step consists in approximating the continuous unknowns $V, p$ or $\xi$ using a finite number of basis functions $\varphi_{i}$, for example $V=\sum_{i} v_{i} \varphi_{i}$. A classical choice is the space $\mathrm{P} 0$, spanned by basis functions $\psi_{i}$ equal to 1 on triangle $T_{i}$ and 0 elsewhere. Another possibility is the space P1, whose basis functions $\phi_{i}$ are equal to 1 on vertex $i, 0$ on all other vertices, and linear on each triangle. If $N_{v}$ (resp. $N_{t}$ ) represents the number of vertices (resp. triangles) in the mesh, the number of P1 (resp. P0) basis functions is $N_{v}$ (resp. $N_{t}$ ). For closed meshes, $N_{t}=2\left(N_{v}-2\right)$. Higher-order basis functions are not useful with meshes composed of flat elements, the additional precision being wasted since the total error of the method becomes dominated by the geometrical error. 


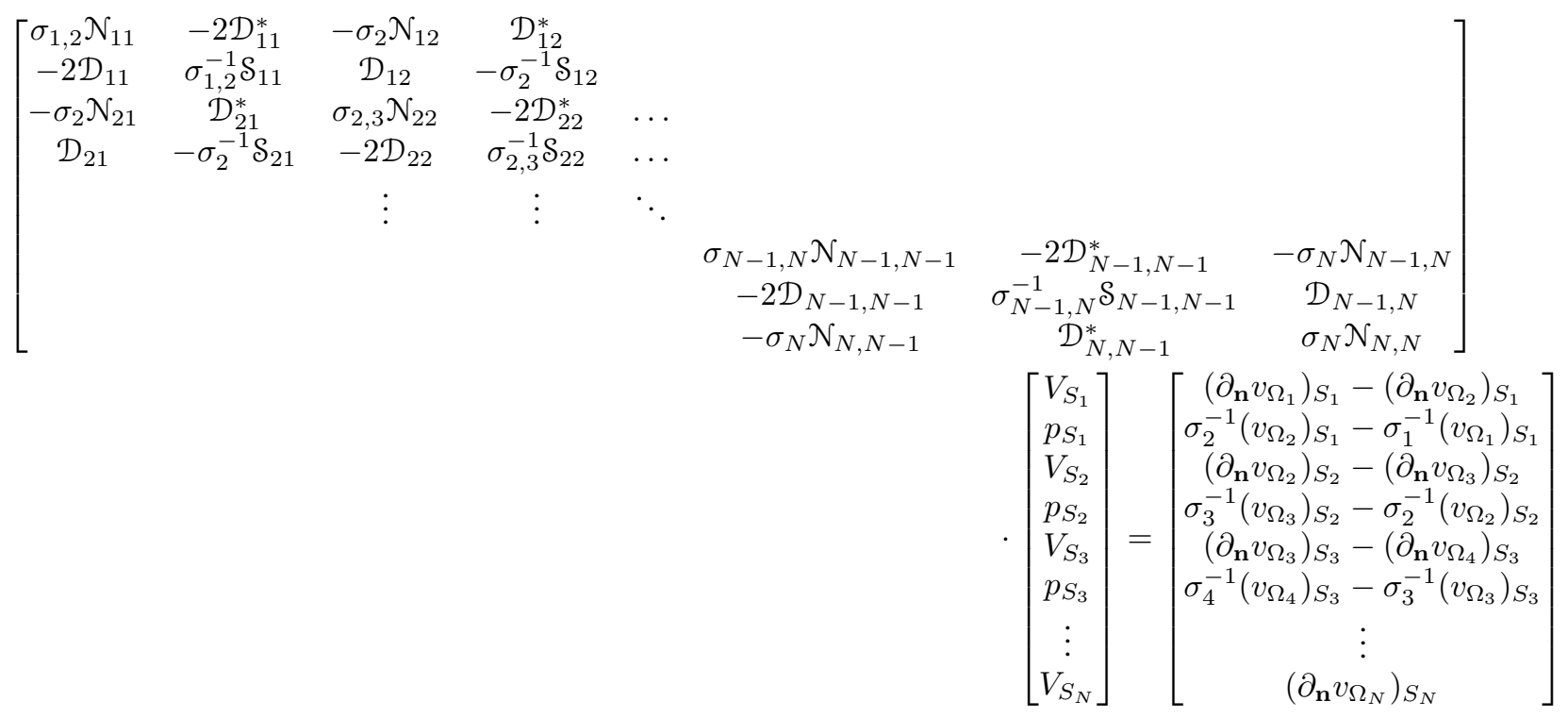

Fig. 4. System representing the continuous operator version of the symmetric method. Observe that the system is symmetric and block-diagonal. Special care is needed in writing the last block because of the conductivity $\sigma_{N+1}=0$. We have noted $\sigma_{i, i+1}$ the sum $\sigma_{i}+\sigma_{i+1}$ and $\sigma_{i, i+1}^{-1}$ the sum $\sigma_{i}^{-1}+\sigma_{i+1}^{-1}$.

\section{Test functions}

Third, to convert the continuous equations of discrete variables into a set of discrete equations, we integrate each of them against a set of test functions $\tilde{\varphi}_{j}$. The simplest choice of test functions is a Dirac mass, $\tilde{\varphi}_{i}=\delta_{\mathbf{x}_{i}}$. This method, called "collocation", is comparatively simple and fast, but often not very accurate. One normally chooses as many collocation points as unknowns. Special care is needed to evaluate the functions at non-regular points of the surface, such as vertices (see Appendix D).

Galerkin-type methods replace the pointwise equality by an equality in the mean sense. The test functions $\tilde{\varphi}_{i}$ are often chosen equal to the basis functions $\varphi_{i}$; this leads to square system matrices. There is an extra integration involved which most of the time needs to be performed numerically. Many times the integrand is singular which augments the difficulty. Galerkin methods are hence more difficult to implement and slower than collocation, but usually more accurate $[8,12$, 20]. We shall therefore concentrate on Galerkin methods in the detailed treatment of the three integral formulations that follows, even though we report the results for the collocation methods as well.

\section{Single-layer formulation}

The continuous equation (15) obtained in the single-layer approach (III-D) is discretized using a Galerkin method, described above. The single-layer density $\xi_{S_{k}}$ on $S_{k}$ is represented as $\xi_{S_{k}}(\mathbf{r})=\sum_{i} x_{i}^{(k)} \varphi_{i}^{(k)}(\mathbf{r})$, where $\varphi_{i}$ can be either a P0 or a P1 basis function. Taking the scalar product of equation (15) (in which $\xi_{S_{k}}$ has been discretized) with the same functions $\varphi_{i}^{(k)}$ yields the following set of equations:

$$
\begin{array}{r}
\left\langle\partial_{\mathbf{n}} v_{s}, \varphi_{i}^{(k)}\right\rangle=\frac{\sigma_{k}+\sigma_{k+1}}{2\left(\sigma_{k+1}-\sigma_{k}\right)}\left(\sum_{j} x_{j}^{(k)}\left\langle\varphi_{i}^{(k)}, \varphi_{j}^{(k)}\right\rangle\right)- \\
\sum_{l=1}^{N} \sum_{j} x_{j}^{(l)}\left\langle\mathcal{D}_{l k}^{*} \varphi_{j}^{(l)}, \varphi_{i}^{(k)}\right\rangle
\end{array}
$$

The explicit matrix form is

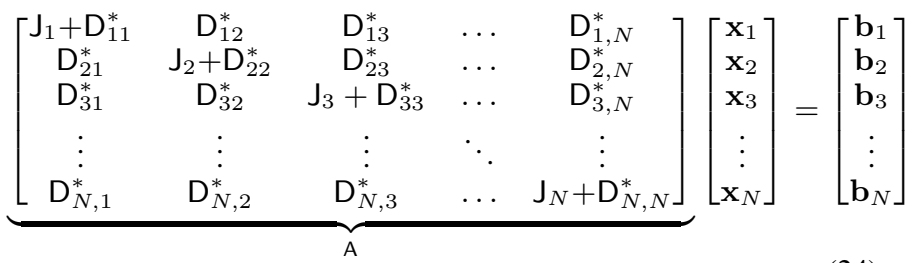

where the matrices $\mathrm{J}$ (which are almost diagonal) are given by

$$
\left(\mathrm{J}_{k}\right)_{i j}=\frac{\sigma_{k}+\sigma_{k+1}}{2\left(\sigma_{k+1}-\sigma_{k}\right)}\left\langle\varphi_{i}^{(k)}, \varphi_{j}^{(k)}\right\rangle
$$

the matrices $D^{*}$ by

$$
\left(\mathrm{D}_{k l}^{*}\right)_{i j}=-\left\langle\mathcal{D}_{k l}^{*} \varphi_{j}^{(l)}, \varphi_{i}^{(k)}\right\rangle
$$

and the vectors $\mathbf{b}$ and $\mathbf{x}$ by

$$
\left(\mathbf{b}_{k}\right)_{i}=\left\langle\partial_{\mathbf{n}} v_{s}, \varphi_{i}^{(k)}\right\rangle, \quad\left(\mathbf{x}_{k}\right)_{i}=x_{i}^{(k)} .
$$

Care is needed in calculating the elements $\left(D_{k k}^{*}\right)_{i i}$ because of the singularity of the operator $\mathcal{D}^{*}$ (see (6)). Some authors adjust the diagonal values to compensate the numerical errors of the rest of the elements using the fact that the sum of the columns of $D_{k k}^{*}$ is known (see [10] for the double-layer approach). This arises from the fact that the total solid angle $\omega=4 \pi\left(\mathcal{D}_{i} 1\right)(\mathbf{r})$ must be equal to $4 \pi$ for all interior points, 
and from the physical necessity of obtaining a singular matrix (see Appendix C). The notation $\mathcal{D}_{i}$ indicates that the operator $\mathcal{D}$ is restricted to the $i$ th interface. However, we prefer to set $\left(\mathrm{D}_{k k}^{*}\right)_{i i}$ to 0 , which is exact at regular points of flat surfaces (triangles), trivial to compute, and unlike the former approach does not obscure potential accuracy problems. We did not observe a significant difference in accuracy between the two choices.

The system matrix $A$ is full and non-symmetric. The elements of the matrices $D^{*}$ involve double integrals over triangles of the meshes. The inner integrals can be calculated analytically for both P0 and P1 basis functions [19, 29, 30]; the outer integrals must be calculated numerically, which is most efficiently done using a Gaussian quadrature adapted to triangles $[6,31]$.

Once $\mathbf{x}$ is known, the potential $V$ is calculated directly from (III-D) as

$V(\mathbf{r})=v_{s}(\mathbf{r})+\sum_{l=1}^{N-1} \sum_{j} x_{j}^{(l)}\left(\mathcal{S}_{l k} \varphi_{j}^{(l)}\right)(\mathbf{r}) \quad$ for $\quad \mathbf{r} \in S_{k}$.

Note that no approximation is involved here; if $\mathbf{x}$ is known exactly, $V$ can be calculated exactly too.

\section{E. Deflation}

An important point to note is that the matrix A presented in (24) is singular (see Appendix C). We "deflate" it [32] using the condition $\langle\xi, 1\rangle=0$ (see Appendix C). For the commonly used basis functions satisfying the partition of unity property $^{3}$, this is equivalent to $\sum_{i} x_{i}^{(k)}=0$ on each $S_{k}$, and thus $\sum_{i k} x_{i}^{(k)}=0$. To impose this, we replace $\mathrm{A}$ with $\mathrm{A}^{\prime}=\mathrm{A}+\omega \mathbf{1 1} \mathbf{1}^{T}$, where $\omega$ is chosen such that $\mathrm{A}^{\prime}$ is well conditioned. The optimal choice of $\omega$ is too costly to calculate but the value is not very critical and can be approximated [12, 33]. We use the fact that $A$ is approximately diagonal dominant and we assume that the very first element is representative, which leads to $\omega=(\mathrm{A})_{11} / M$, where $M$ is the total number of unknowns. This was found to perform acceptably well. The deflated matrix $A^{\prime}$ is regular and square and can be inverted by the usual methods.

Note that deflation is not equivalent to regularization that looks for a smooth solution only approximately satisfying the Maxwell equations. Instead, deflation chooses one solution from a family of equivalent ones, all satisfying the equations exactly, according to our preferences based on the physics of the problem.

\section{F. Double-layer formulation}

The double-layer formulation (16) is discretized using the same approach as the single-layer one, with $V_{S_{k}}$ on $S_{k}$ represented as $V_{S_{k}}(\mathbf{r})=\sum_{i} x_{i}^{(k)} \varphi_{i}^{(k)}(\mathbf{r})$, where $\varphi_{i}$ is either

\footnotetext{
${ }^{3}$ Their sum is equal to 1 everywhere.
}

P0 or P1. Taking the scalar product of (16) with $\varphi_{i}^{(k)}$ yields

$$
\begin{gathered}
\left\langle v_{d}, \varphi_{i}^{(k)}\right\rangle=\frac{\sigma_{k}+\sigma_{k+1}}{2}\left(\sum_{j} x_{j}^{(k)}\left\langle\varphi_{i}^{(k)}, \varphi_{j}^{(k)}\right\rangle\right)- \\
\sum_{l=1}^{N}\left(\sigma_{l+1}-\sigma_{l}\right) \sum_{j} x_{j}^{(l)}\left\langle\mathcal{D}_{k l} \varphi_{j}^{(l)}, \varphi_{i}^{(k)}\right\rangle
\end{gathered}
$$

or, in a matrix form

$$
\underbrace{\left[\begin{array}{ccccc}
\mathrm{J}_{1}+\mathrm{D}_{11} & \mathrm{D}_{12} & \mathrm{D}_{13} & \ldots & \mathrm{D}_{1, N} \\
\mathrm{D}_{21} & \mathrm{~J}_{2}+\mathrm{D}_{22} & \mathrm{D}_{23} & \ldots & \mathrm{D}_{2, N} \\
\mathrm{D}_{31} & \mathrm{D}_{32} & \mathrm{~J}_{3}+\mathrm{D}_{33} & \ldots & \mathrm{D}_{3, N} \\
\vdots & \vdots & \vdots & \ddots & \vdots \\
\mathrm{D}_{N, 1} & \mathrm{D}_{N, 2} & \mathrm{D}_{N, 3} & \ldots & \mathrm{J}_{N}+\mathrm{D}_{N, N}
\end{array}\right]}_{\mathrm{A}}\left[\begin{array}{c}
\mathbf{x}_{1} \\
\mathbf{x}_{2} \\
\mathbf{x}_{3} \\
\vdots \\
\mathbf{x}_{N}
\end{array}\right]=\left[\begin{array}{c}
\mathbf{b}_{1} \\
\mathbf{b}_{2} \\
\mathbf{b}_{3} \\
\ldots \\
\mathbf{b}_{N}
\end{array}\right]
$$

where

$$
\begin{aligned}
\left(\mathrm{J}_{k}\right)_{i j} & =\frac{\sigma_{k}+\sigma_{k+1}}{2}\left\langle\varphi_{i}^{(k)}, \varphi_{j}^{(l)}\right\rangle \\
\left(\mathrm{D}_{k l}\right)_{i j} & =-\left(\sigma_{l+1}-\sigma_{l}\right)\left\langle\mathcal{D}_{k l} \varphi_{j}^{(l)}, \varphi_{i}^{(k)}\right\rangle \\
\left(\mathbf{b}_{k}\right)_{i} & =\left\langle v_{d}, \varphi_{i}^{(l)}\right\rangle, \quad\left(\mathbf{x}_{k}\right)_{i}=x_{i}^{(k)}
\end{aligned}
$$

As in the single-layer case, and thanks to the duality between $\mathcal{D}$ and $\mathcal{D}^{*}$, the inner integrals needed to calculate elements of matrices $D_{l k}$ have an analytical solution for both P0 and P1 basis functions [19,29], while the outer integrals are calculated numerically [8]. The matrix is again full, nonsymmetric, and needs to be deflated, this time because the potential $V$ is only defined up to a constant (see Appendix C). Imposing the condition $\mathscr{H}$ is impractical, and we instead impose either the mean of the potential over all surfaces to be zero, $\sum_{k=1}^{N} \sum_{i} x_{i}^{(k)}=0$, or else the mean of the potential over the external surface to be zero, $\sum_{i} x_{i}^{(N)}=0$. In the latter case we propose to modify (deflate) only the bottomright block of $\mathrm{A}$, namely $\mathrm{J}_{N}+\mathrm{D}_{N, N}$. The basis functions are assumed to satisfy the partition of unity property.

The continuous $V$ is directly accessible from the discretization equation $V(\mathbf{r})=\sum_{i} x_{i}^{(k)} \varphi_{i}^{(k)}$ for $\mathbf{r} \in S_{k}$.

\section{G. Symmetric approach}

The specificity of the discretization of the symmetric approach $(20,21)$ is that both $V$ and its derivative $p$ are simultaneously involved as unknowns. The approximation errors for the two quantities should be asymptotically equivalent, so that the overall error is not dominated by either one. For this reason, we choose to approximate $V$ using P1 basis functions as $V_{S_{k}}(\mathbf{r})=\sum_{i} x_{i}^{(k)} \phi_{i}^{(k)}(\mathbf{r})$, while its derivative $p$ is represented using the space $\mathrm{P} 0, p_{S_{k}}(\mathbf{r})=\sum_{i} y_{i}^{(k)} \psi_{i}^{(k)}(\mathbf{r})$. Similar concerns guide our choice of test functions. We notice that the operator $\mathcal{S}$ behaves as a smoother: it increases the regularity of its argument [7] by one. The operators $\mathcal{D}, \mathcal{D}^{*}$ do not change it, while $\mathcal{N}$ has a derivative character: it decreases the regularity by one. The regularity is closely tied to an approximation order [34]. To balance the errors, all the scalar products should have the same approximation order. To ensure 
this, we multiply the equation (20) for the potential (a P1 function) by $\mathrm{P} 0$ test functions $\psi_{i}$

$$
\begin{gathered}
\left\langle\sigma_{k+1}^{-1} v_{\Omega_{k+1}}-\sigma_{k}^{-1} v_{\Omega_{k}}, \psi_{i}^{(k)}\right\rangle= \\
\sum_{j} x_{j}^{(k-1)}\left\langle\mathcal{D}_{k, k-1} \phi_{j}^{(k-1)}, \psi_{i}^{(k)}\right\rangle+ \\
\sum_{j} x_{j}^{(k+1)}\left\langle\mathcal{D}_{k, k+1} \phi_{j}^{(k+1)}, \psi_{i}^{(k)}\right\rangle+ \\
\left(\sigma_{k}^{-1}+\sigma_{k+1}^{-1}\right) \sum_{j} y_{j}^{(k)}\left\langle\mathcal{S}_{k k} \psi_{j}^{(k)}, \psi_{i}^{(k)}\right\rangle- \\
\sigma_{k}^{-1} \sum_{j} y_{j}^{(k-1)}\left\langle\mathcal{S}_{k, k-1} \psi_{j}^{(k-1)}, \psi_{i}^{(k)}\right\rangle- \\
\sigma_{k+1}^{-1} \sum_{j} y_{j}^{(k+1)}\left\langle\mathcal{S}_{k, k+1} \psi_{j}^{(k+1)}, \psi_{i}^{(k)}\right\rangle- \\
2 \sum_{j} x_{j}^{(k)}\left\langle\mathcal{D}_{k k} \phi_{j}^{(k)}, \psi_{i}^{(k)}\right\rangle
\end{gathered}
$$

and the equation (21) for the flow (a P0 function) by P1 test functions $\phi_{i}$

$$
\begin{aligned}
& \left\langle\partial_{\mathbf{n}} v_{\Omega_{k+1}}-\partial_{\mathbf{n}} v_{\Omega_{k}}, \phi_{i}^{(k)}\right\rangle= \\
& \sigma_{k} \sum_{j} x_{j}^{(k-1)}\left\langle\mathcal{N}_{k, k-1} \phi_{j}^{(k-1)}, \phi_{i}^{(k)}\right\rangle+ \\
& \sigma_{k+1} \sum_{j} x_{j}^{(k+1)}\left\langle\mathcal{N}_{k, k+1} \phi_{j}^{(k+1)}, \phi_{i}^{(k)}\right\rangle- \\
& \left(\sigma_{k}+\sigma_{k+1}\right) \sum_{j} x_{j}^{(k)}\left\langle\mathcal{N}_{k k} \phi_{j}^{(k)}, \phi_{i}^{(k)}\right\rangle- \\
& \sum_{j} y_{j}^{(k-1)}\left\langle\mathcal{D}_{k, k-1}^{*} \psi_{j}^{(k-1)}, \phi_{i}^{(k)}\right\rangle- \\
& \sum_{j} y_{j}^{(k+1)}\left\langle\mathcal{D}_{k, k+1}^{*} \psi_{j}^{(k+1)}, \phi_{i}^{(k)}\right\rangle+ \\
& 2 \sum_{j} y_{j}^{(k)}\left\langle\mathcal{D}_{k k}^{*} \psi_{j}^{(k)}, \phi_{i}^{(k)}\right\rangle
\end{aligned}
$$

both to hold on all interfaces $k=1, \ldots, N$. This set of equations can be expressed more concisely in matrix form The matrix A should be truncated ${ }^{4}$ like in (22), to account for the zero conductivity $\sigma_{N+1}=0$.

Note that $A$ is larger than in the single or double-layer cases. However, it is symmetric and block-diagonal, which means that the actual number of elements to be stored is comparable or even smaller, depending on the number of interfaces. Moreover, matrices $\mathrm{N}_{k l}$ can be calculated at negligible costs from the intermediate results needed for calculating matrices $S_{k l}$, thanks to an interesting relation coming from Theorem 3.3.2 in [7]:

$$
\left\langle\mathcal{N}_{k l} \phi_{i}^{\prime}, \phi_{j}^{\prime}\right\rangle=-\left(\mathbf{q}_{i} \times \mathbf{n}_{i}\right)\left(\mathbf{q}_{j} \times \mathbf{n}_{j}\right)\left\langle\mathcal{S}_{k l} \psi_{j}^{(l)}, \psi_{i}^{(k)}\right\rangle
$$

where $\varphi_{i}^{\prime}(\mathbf{x})=\left(\mathbf{q}_{i} \cdot \mathbf{x}+\alpha_{i}\right) \psi_{i}(\mathbf{x})$ and $\varphi_{j}^{\prime}(\mathbf{x})=\left(\mathbf{q}_{j}\right.$. $\left.\mathbf{x}+\alpha_{j}\right) \psi_{j}(\mathbf{x})$ are the $\mathbf{P} 1$ basis functions $\varphi$ restricted to one triangle.

Deflation is needed to avoid the indetermination of $V$. To impose a zero mean of the potential on the outermost surface, only the bottom-right block with $\mathrm{N}_{N, N}$ is modified

\footnotetext{
${ }^{4}$ The bottom-right corner of $\mathrm{A}$ is not shown here for space reasons.
}

TABLE I

THE DIFFERENT METHODS IMPLEMENTED AND THEIR ASSOCIATED LABELS.

\begin{tabular}{|c|c|c|c|}
\hline Label & Formulation & $\varphi$ & $\psi$ \\
\hline \hline $1 \mathrm{a}$ & Single-Layer & P0 & Dirac \\
$1 \mathrm{~b}$ & & P0 & P0 \\
1c & & P1 & P1 \\
\hline 2a & Double-Layer & P0 & Dirac \\
2b & & P0 & P0 \\
2c & & P1 & P1 \\
\hline 3 & Symmetric & P0 & P1 \\
\hline
\end{tabular}

to $\mathrm{N}_{N, N}+\omega \mathbf{1 1}^{T}$, using the heuristic $\omega=\left(\mathrm{N}_{N, N}\right)_{11} / M_{N}$, as in Section IV-E.

\section{H. Acceleration}

As the number of mesh elements $M$ grows, the matrix assembly time $O\left(M^{2}\right)$ becomes dominated by the time needed to solve the resulting linear system $O\left(M^{3}\right)$, e.g. by the LU decomposition. Iterative solvers $[8,9,35]$ can be used instead, reducing the computation time and only accessing the matrix by matrix-vector multiplications Az. This brings other optimization opportunities such as calculating these products approximately using a fast multipole method (FMM) [11], precorrected-FFT $[14,36]$ or SVD-based methods. Multiresolution techniques permit to reduce the number of expensive iterations on the finest level by solving first a reduced size problem and using its solution as the starting guess. Multigrid algorithms combine iterations on fine and coarse levels for even faster convergence.

Parallelizing the assembly phase is straightforward as the matrix elements can be calculated independently, even though for optimum performance the expensive calculations needed to calculate $S$ should be reused for the calculation of $N$, as mentioned above. Parallel techniques also exist for linear system solver non-iterative algorithms (SCALAPACK library).

\section{EXPERIMENTS}

We have implemented the single-layer, double-layer, and symmetric approaches described in this article in both serial and parallel versions. The single and double-layer approaches exist in three discretization variants: with the collocation method $\left(\tilde{\varphi}_{j}=\delta_{\mathbf{x}_{j}}\right)$ using the P0 basis functions $\varphi$, and with the Galerkin method $\left(\tilde{\varphi}_{j}=\varphi_{j}\right)$ using both P0 and P1 bases. The symmetric method is discretized using P1 basis functions for $V$ and $\mathrm{P} 0$ basis functions for $p$. We have implemented only this choice, since with other discretizations we loose the principal advantages of the method, symmetry and accuracy. We have applied these methods first to synthetic cases where an analytical solution is known, as well as to realistically shaped head models.

Table I summarizes the different discretization choices, and indicates the labels by which they are referenced in the text and figures. 


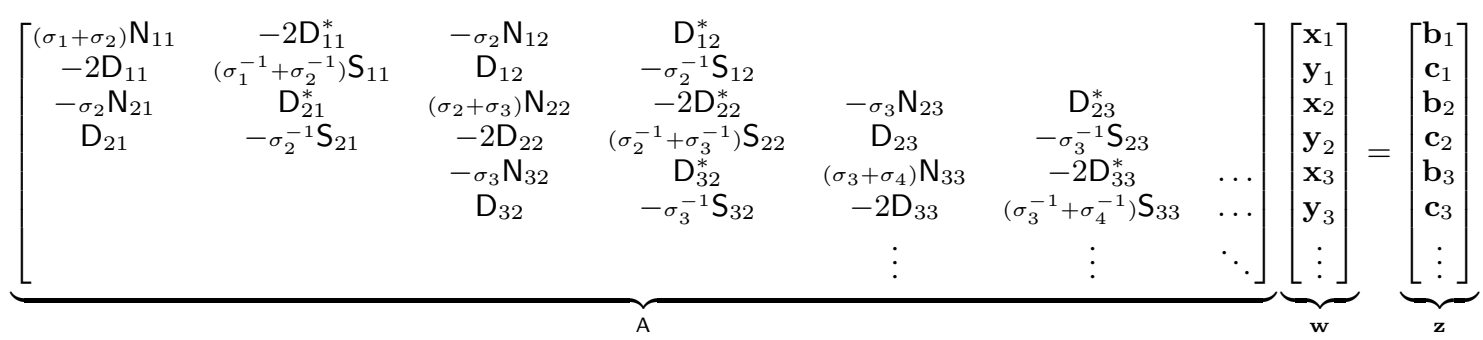

with

$$
\begin{aligned}
\left(\mathrm{N}_{k l}\right)_{i j} & =\left\langle\mathcal{N}_{k l} \phi_{j}^{(l)}, \phi_{i}^{(k)}\right\rangle \\
\left(\mathrm{D}_{k l}\right)_{i j} & =\left(\mathrm{D}_{l k}^{*}\right)_{j i}=\left\langle\mathcal{D}_{k l} \phi_{j}^{(l)}, \psi_{i}^{(k)}\right\rangle \\
\left(\mathbf{b}_{k}\right)_{i} & =\left\langle\partial_{\mathbf{n}} v_{\Omega_{k}}-\partial_{\mathbf{n}} v_{\Omega_{k+1}}, \phi_{i}^{(k)}\right\rangle \\
\left(\mathbf{x}_{k}\right)_{i} & =x_{i}^{(k)}
\end{aligned}
$$

\section{A. Speed}

The speed depends strongly on the desired precision, on the optimization and acceleration techniques applied and on the specific task. ${ }^{5}$ In our experiments, the time needed for the direct assembly of the matrix was of the order of 1 to $5 \mathrm{~s}$ for our smallest head mesh of $3 \times 42$ vertices, to several minutes to assemble the matrices of $4486 \times 4486$ elements, corresponding to the meshes of $3 \times 642$ vertices, up to about $2 \mathrm{~h}$ for the matrices of $17926 \times 17926$, corresponding to meshes of $3 \times 2562$ vertices. Using a parallel code on a cluster of workstations speeds the assembling proportionally to the number of processors with a high efficiency. The time needed to solve the linear system of equations varied between $10 \mathrm{~ms}$ and $2 \mathrm{~h}$ for the same cases. Generally, the assembly time grows quadratically with the number of degrees of freedom, and the solution time as a third power. Collocation methods can be 10 or more times faster than the Galerkin method, depending on the numerical integration method used and the number of integration points needed to get the required accuracy. The single-layer method is more costly than the corresponding double-layer method ${ }^{6}$, as two matrices need to be assembled, the matrix $A$ in (24) in order to solve for the single-layer density, and an additional matrix in order to integrate the potential from equation (25).

\section{B. Spherical head models}

The first part of our tests was performed on triangulated spherical surfaces. The choice of a spherical geometry has the advantage that an analytical solution is available [20,37, 38 ], thus making it possible to evaluate the accuracy of the different methods. The spherical surfaces were triangulated with progressively finer meshes of 42,162 , and 642 vertices per layer ${ }^{7}$. We used three concentric spheres with radii 0.87 ,

\footnotetext{
${ }^{5}$ For example, one may consider that the system matrix, once assembled, can be used to solve many problems involving the same geometry. This makes the actual assembly time irrelevant.

${ }^{6}$ The precise ratio depends on the discretization used.

${ }^{7}$ Finer meshes were avoided in this set of experiments because of memory and time limitations so that a serial direct solver could be used for most reproducible results.
}

$$
\begin{aligned}
\left(\mathrm{S}_{k l}\right)_{i j} & =\left\langle\mathcal{S}_{k l} \psi_{j}^{(l)}, \psi_{i}^{(k)}\right\rangle \\
\left(\mathbf{c}_{k}\right)_{i} & =\left\langle\sigma_{k+1}^{-1} v_{\Omega_{k+1}}-\sigma_{k}^{-1} v_{\Omega_{k}}, \psi_{i}^{(k)}\right\rangle \\
\left(\mathbf{y}_{k}\right)_{i} & =y_{i}^{(k)}
\end{aligned}
$$

0.92 , and 1.0, delimiting volumes with conductivities 1.0, $0.0125,1.0$ and 0.0 , from inside towards outside. The sources were unitary current dipoles oriented as $\left[\begin{array}{lll}1 & 0 & 1\end{array}\right] / \sqrt{2}$ and placed at distances $r=0.425,0.68,0.765,0.8075$, and 0.8415 from the center on the $x$ axis.

We chose to evaluate the analytical solution at triangle centers for the P0 methods and at vertex points for the others. This disadvantages Galerkin methods but it is close to actual use. We then calculated the relative $\ell_{2}$ error $\| \mathbf{v}_{\text {anal }}-$ $\mathbf{v}_{\text {num }}\left\|_{\ell_{2}} /\right\| \mathbf{v}_{\text {anal }} \|_{\ell_{2}}$, making sure that both $\mathbf{v}_{\text {anal }}$ and $\mathbf{v}_{\text {num }}$ had zero means prior to comparison. Note that some authors linearly scale $\mathbf{v}_{\text {num }}$ to obtain the best fit [10]. This obviously significantly reduces the reported error but is difficult to justify in the context of evaluating the accuracy of a method.

We have also made some experiments using a single sphere model, not shown here because of lack of space and because it does not correspond to a plausible head model. Note that in this case the symmetric model is disadvantaged by using only the operator $\mathcal{N}$.

1) Error versus dipole position: The first set of experiments (Fig. 5) shows how the accuracy decreases when the current dipole source approaches the surface of discontinuity. We observe that the symmetric approach is much less affected than the other methods.

2) Error versus mesh density: For a fixed source position $(r=0.765)$, the error decreases as the mesh is refined (Fig. 6). We observe that while both collocation variants produce the largest errors (results are completely unreliable), Galerkin methods based on P0 approximations are better, and the best results are provided by the P1 methods, namely by the symmetric formulation. Moreover, the slope of the decrease of the error with mesh size is steeper for P1-based methods, a benefit of their higher approximation order.

3) Error versus conductivity: The accuracy of all implemented methods depends on the ratio of conductivities between the second layer (representing the skull) and the neighboring volumes (representing brain and scalp). To display this behavior, we have created additional head models with conductivities of the three volumes $1.0, \sigma, 1.0$, with $\sigma$ ranging between $1 / 2$ and $1 / 1000$. Figure 7 shows that when the con- 


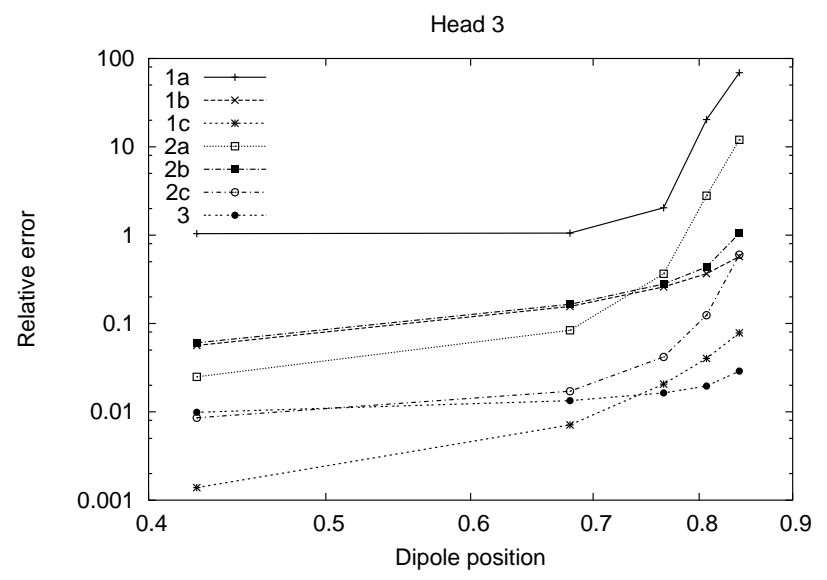

Fig. 5. The relative error versus the dipole position $r$ for meshes with 642 vertices per sphere. The label 1 (resp. 2) refers to single-layer (resp. double-layer) potential, and the label 3 refers to the symmetric formulation, as explained in Table I.

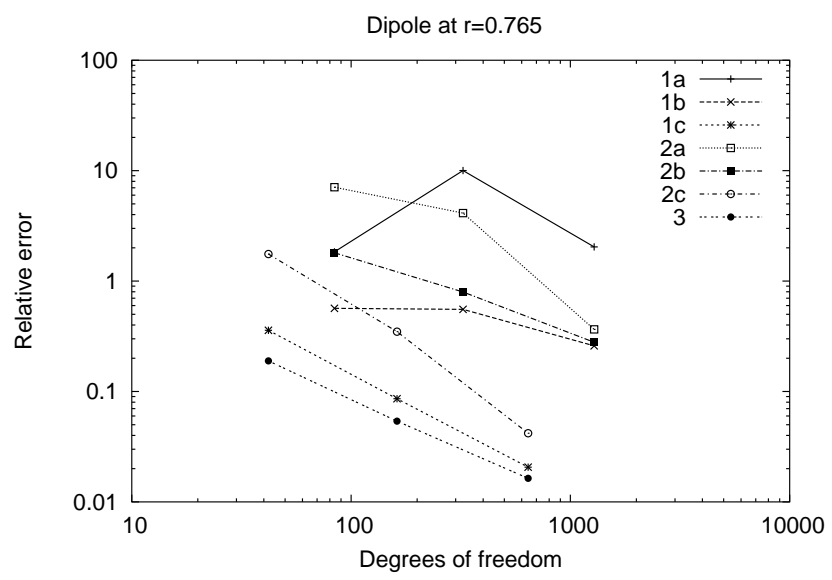

Fig. 6. The relative error versus the number of the degrees of freedom of the solution for the dipole source at $r=0.765$. Refer to Table I for the meaning of the labels. Both P0 collocation methods in the three-sphere case are outside their area of applicability and do not provide meaningful results.

ductivity ratio $\sigma$ becomes small, the precision of the single and double-layer methods drops. The symmetric method displays a clear advantage over the others since, on the contrary, its accuracy increases as $\sigma \rightarrow 0$. This is a valuable result in the context of human head modelling where $\sigma \approx 0.01$.

\section{Realistic meshes}

We generated a realistic four-layer model of the head with about 13000 points and 26000 faces $^{8}$. Figure 8 shows the potential field created by a dipole close to a cortex surface using a parallel implementation of our algorithm. This gives an indication of the complexity of the model we are currently able to process. The cortex surface contains 4960 points and the average size of the triangles' edges is $5 \mathrm{~mm}$. The simulations have been performed with a dipole located inside at roughly $10 \mathrm{~mm}$ from this surface. The total computation took close to

\footnotetext{
${ }^{8}$ We thank Geoffray Adde and Florent Segonne for preparing the hierarchical meshes from the MRI data.
}

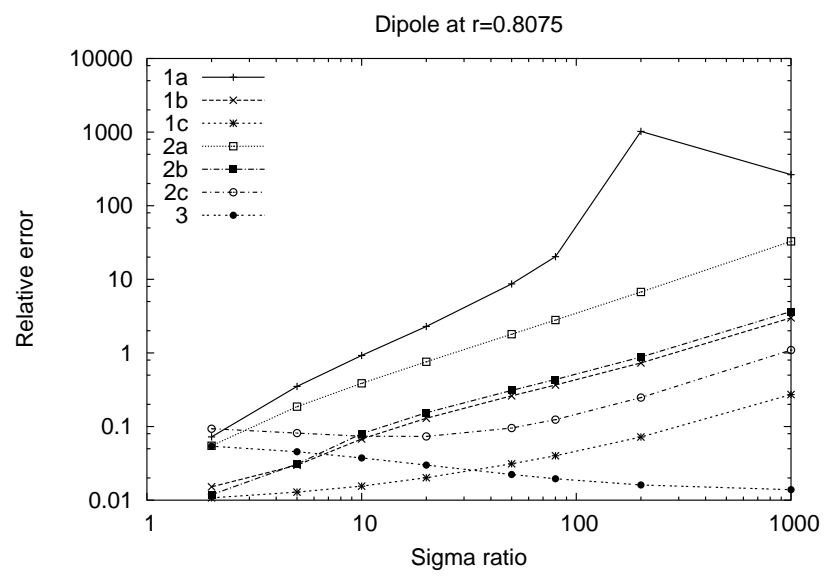

Fig. 7. The relative error versus the ratio of conductivities between neighboring layers, for dipole at $r=0.8075$ and mesh of 642 vertices. Refer to Table I for the meaning of the labels.

6 hours on a cluster of 30 PCs. It required of the order of 1000 iterations.

We used decimated versions of the same head model to compare the time and memory requirements of the different methods in this realistic setting. We had to make several simplifications in order to make this comparison possible. We only included P1 discretizations of the single and doublelayer methods because they are the most comparable with the symmetric variant in terms of representation. The superior accuracy of the Galerkin method for a majority of applications is widely recognized $[8,12,20]$. In order to obtain reliable timing results, we chose to use the sequential ${ }^{9}$ direct $^{10}$ solver, which consequently limits the maximum mesh sizes we could process. Even so, the timing results fluctuate widely ${ }^{11}$ and should only be regarded as indicative. Regarding accuracy comparisons, as we had no way of knowing the ground truth solution, we arbitrarily picked one of the three methods (the double-layer) and used its results at the finest mesh as a reference. Results at other resolutions were interpolated on the finest mesh, and the relative $\ell_{2}$ norm $\left\|\mathbf{x}-\mathbf{x}_{\text {ref }}\right\| /\left\|\mathbf{x}_{\text {ref }}\right\|$ was used to measure accuracy (see Table II) .

In order for the comparison to be meaningful, given that the reference solution was picked as one of the three solutions for the finest discretization, we had to ensure that the other two solutions were very close to that reference, otherwise there would be no reason to believe that the so-called reference should have any relationship with the true solution. The mesh size had to be increased until this requirement was met, and this proved to be difficult because of the mesh size restriction imposed by the sequential direct solver. We had to reduce the number of layers in the model, from four down to two, to increase the separation between layers, and to use a deep

\footnotetext{
${ }^{9}$ The timings on the cluster of PCs vary widely because we have no control over the assignment of our task to different processors and over the load imposed by other users.

${ }^{10}$ The number of iterations of the iterative method varies strongly with the stopping threshold, conditioning of the matrix, and other similar effects.

${ }^{11}$ Most likely because of the virtual memory effect. We have used the total elapsed time ("wall time") as opposed to the "CPU time" as the later seemed to be less relevant.
} 
dipole. At convergence, the results obtained by the three methods differed in relative $\ell_{2}$ norm by roughly $0.05(5 \%)$ for the two-layer model.

We also ran computations on a three-layer model, but in that case, the relative errors between results at the finest discretization were as high as $20 \%$. It would have required even finer meshes to achieve the same relative accuracy as in the two-layer case and it was not possible for the reasons indicated above. This is the reason why the accuracy results in Table II (bottom) compare each method to its own result at the finest discretization.

\section{Discussion}

A meaningful comparison of the various techniques is difficult. First, one must bear in mind that they use different number of degrees of freedom to perform the calculations, which moreover is not necessarily identical to the number of degrees of freedom used to express the solution. More specifically, for a (closed) mesh with $N_{v}$ vertices, P1 methods involve $N_{v}$ unknowns, P0 based methods use about $2 N_{v}$ of them, while the symmetric method with P0/P1 discretization uses about $3 N_{v}$ unknowns, but only $N_{v}$ degrees of freedom to express the solution $V$.

In the case of the spherical models with three layers where the ground truth is known analytically, Fig. 5 shows that for a dipole far from an interface, the single-layer method performs best. This probably comes from the fact that it represents the solution as an exact term plus a correction. When the dipole moves closer to the interface, the symmetric method yields the best results. Fig. 6 shows that the symmetric method is less sensitive than the single-layer and the doublelayer method to a decrease in the quality of the description of the geometry. Finally, Fig. 7 indicates that when the ratio of conductivities between neighboring layers is between roughly 2 and 10 the single-layer method performs best, followed by the symmetric and the traditional double-layer methods, while when this ratio grows larger than 15 , the new symmetric method is clearly ahead of the single- and doublelayer methods.

As stated above, in the case of the much more realistic model of Fig. 8 the comparison between the methods is more difficult since the ground-truth data is unavailable. Nevertheless, all three methods were implemented within the same programming framework, using the same basic functional blocks, and we can learn a number of things from the results shown in Table II (top). It is interesting to note that the symmetric method is less computationally demanding than the alternatives. As already mentioned, this comes from the fact that many of the computations to assemble the system matrix of the symmetric method can be reused, which does not happen for the other methods. Also, integrating the D elements in the symmetric method with P0 and P1 basis functions is easier (the integrand is easier to evaluate) than integrating with respect to two $\mathrm{P} 1$ functions, as it happens in the $\mathrm{D}$ elements for the single and double-layer P1 implementation. The matrix factorization is slower for the symmetric method due to its larger matrix size. However, for the number of unknowns considered this is compensated by the fast assembly.
As far as accuracy is concerned, we see from the last three columns of Table II (top) that when the number of vertices decreases from 2000 to 200 , the performance of all three methods degrade similarly. The symmetric method outperforms the others for coarse meshes, despite of being disadvantaged because the double-layer method was chosen as a reference. The accuracy results for three-layer realistic meshes (Table II (bottom)) indicate a good convergence trend of each method, even though, as we have seen, still higher resolution meshes would be needed to make all of them converge to a common solution. This only highlights the difficulties with solving the EEG forward problem on rough (realistic) surfaces and supports our claim that much finer meshes will have to be used.

\section{CONCLUSION}

We have presented a conceptual framework for Boundary Element Methods in EEG which is based on a theorem (Theorem 1) that characterizes harmonic functions defined on the complement of a bounded smooth surface. This theorem has allowed us to cast the previous approaches in a unified setting and to develop two new approaches corresponding to different ways of looking at the same theorem. Specifically, we have shown that the classical integral formulation that has been used during the last thirty years for EEG and MEG calculations by the BEM and is based on a double-layer potential is not the only possible. We have developed a dual approach which involves a single-layer potential and proposed a symmetric formulation, which combines single- and doublelayer potentials, and is new to the field of EEG, although it has been applied to other problems in electromagnetism $[23,24]$. The three methods have been evaluated numerically using a spherical geometry with known analytical solution, and the symmetric formulation achieves a significantly higher accuracy than the alternative methods. Interestingly enough, the next best method does not seem to be the "traditional" double-layer method but rather the dual single-layer approach. Additionally, we have presented results with realistically shaped meshes. Beside providing a better understanding of the theoretical foundations of BEM, our approach appears to lead also to more efficient algorithms.

It is appealing by its symmetry and its superior accuracy in semi-realistic geometry. The precise theoretical analysis of the accuracy performance of the different methods is difficult because of the number of factors involved and remains to be done, although some partial results can be found in [7, 25, 39].

The main benefit of using the proposed approach is that the error increases much less dramatically when the current sources approach a surface where the conductivity is discontinuous. This implies that we are able to reduce the number of mesh elements in a usable model of the human cortex with a realistic geometry [40]. This has the effect of bringing the idea of accurate electromagnetic simulation of the human brain much closer to what can be achieved with today's technology. Nevertheless, advanced acceleration techniques will still have to be used both at the algorithm and implementation levels before this idea can be really instantiated $[11,41]$. 

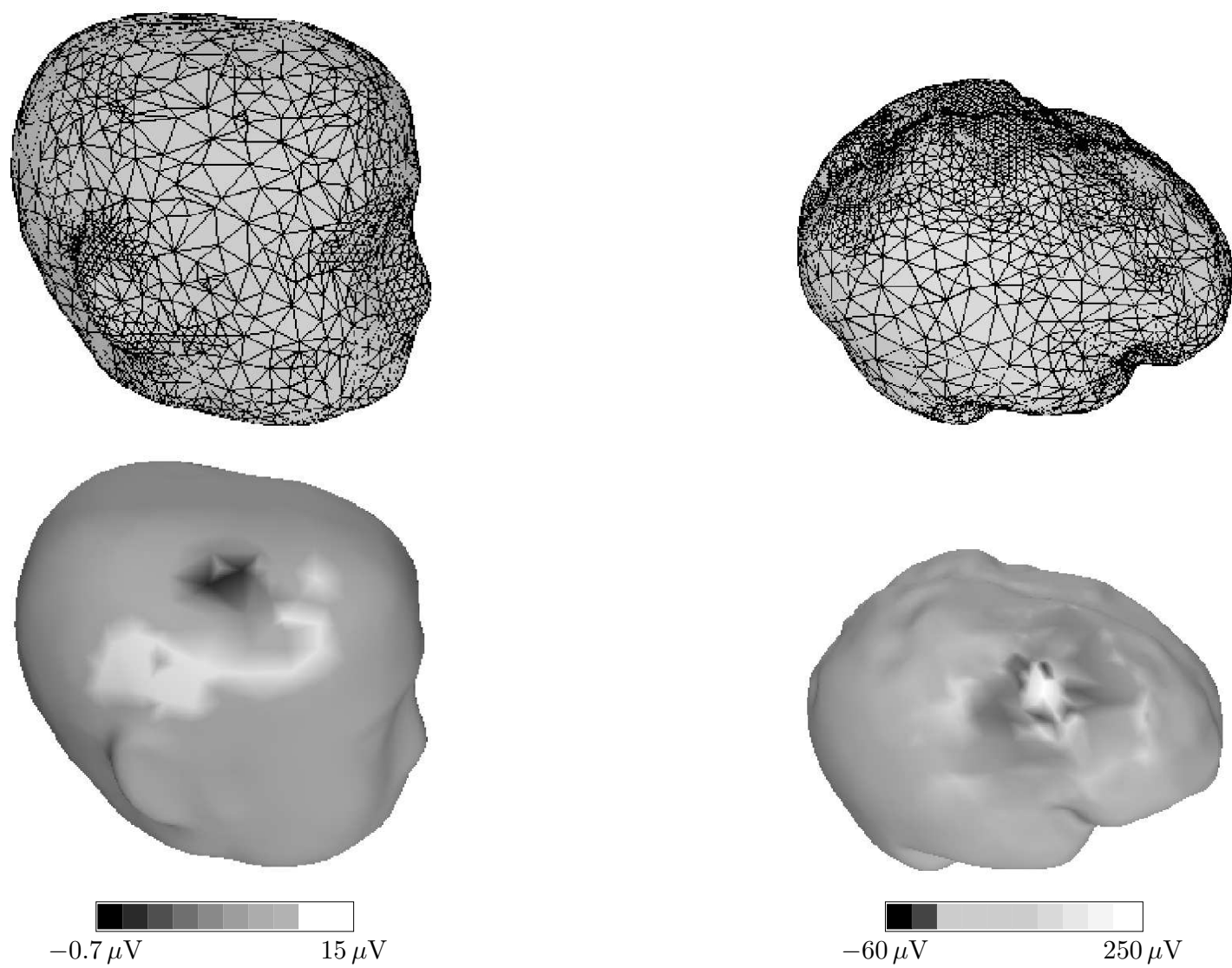

Fig. 8. Top: We have used a realistic four-layer model of the head with about 13000 points and 26000 faces. We show the surfaces corresponding to the skin (left) and to the cortex (right). Bottom: We have calculated the potential fi eld of a dipole close to a cortex surface using a parallel implementation of our algorithm. We show the electric potential on the skin (left) and on the cortex (right).

Future work includes a better understanding of the accuracy improvements and extensions of the method.

\section{APPENDIX}

We recall some basic identities between surface and volume integrals involving vector fields, leading to the Representation Theorem 1. Note that in this appendix we use an explicit integral notation for didactic purposes, while in the body of the article we have privileged the conciseness of the operator notation (6).

\section{A. Representation Theorems}

Consider a simplified version of Problem (1): the Poisson problem $\Delta u=f$. It is well-known that the so-called Green function (5) is its fundamental solution, i.e. $-\Delta G=\delta_{0}$ in $\mathbb{R}^{3}$ in the distributional sense, where $\delta_{0}$ is the Dirac mass at the origin. By translation invariance, we have

$$
-\Delta_{\mathbf{r}} G\left(\mathbf{r}-\mathbf{r}^{\prime}\right)=\delta_{0}\left(\mathbf{r}-\mathbf{r}^{\prime}\right)=\delta_{\mathbf{r}^{\prime}},
$$

where the notation $\Delta_{\mathrm{r}}$ signifies that partial derivatives are taken with respect to the variable $\mathbf{r}$, and $\delta_{\mathbf{r}^{\prime}}$ is a Dirac mass centered at $\mathbf{r}^{\prime}$.

There are many fundamental solutions to the Poisson problem, but the Green function (5) is the only one with radial symmetry (a function of the radius $r=\|\mathbf{r}\|$ ) and vanishing at infinity $(r \rightarrow \infty)$.

Given a bounded and compact open set $\Omega \subseteq \mathbb{R}^{3}$ with a regular boundary $\partial \Omega$ which may not be connected, the divergence theorem $\int_{\Omega} \nabla \cdot \mathbf{g}\left(\mathbf{r}^{\prime}\right) \mathrm{d} \mathbf{r}^{\prime}=\int_{\partial \Omega} \mathbf{g}\left(\mathbf{r}^{\prime}\right) \cdot \mathrm{d} \mathbf{s}\left(\mathbf{r}^{\prime}\right)$, where $\mathrm{d} \mathbf{s}\left(\mathbf{r}^{\prime}\right)=\mathbf{n}^{\prime}\left(\mathbf{r}^{\prime}\right) \mathrm{d} s\left(\mathbf{r}^{\prime}\right)$, relates the integral over a volume $\Omega$ with a surface integral over its boundary $\partial \Omega$. For scalar distributions $u, v$, substituting $\mathbf{g}=u \nabla v$ yields the first Green identity $\int_{\partial \Omega} u \nabla v \cdot \mathrm{d} \mathbf{s}\left(\mathbf{r}^{\prime}\right)=\int_{\Omega} \nabla u \cdot \nabla v+u \Delta v \mathrm{~d} \mathbf{r}^{\prime}$. Exchanging $u, v$ and subtracting the resulting equations gives the second ${ }^{12}$ Green identity [42]

$$
\begin{aligned}
\int_{\Omega} u \Delta v-v \Delta u \mathrm{~d} \mathbf{r}^{\prime} & =\int_{\partial \Omega}(u \nabla v-v \nabla u) \cdot \mathrm{d} \mathbf{s}\left(\mathbf{r}^{\prime}\right) \\
& =\int_{\partial \Omega} u \partial_{\mathbf{n}^{\prime}} v-v \partial_{\mathbf{n}^{\prime}} u \mathrm{~d} s\left(\mathbf{r}^{\prime}\right)
\end{aligned}
$$

where $\mathbf{n}$ is normal to $\partial \Omega$, pointing outward (from $\Omega$ to its complement $\Omega^{c} \equiv \mathbb{R}^{3} \backslash \bar{\Omega}$ ), and is denoted $\mathbf{n}^{\prime}$ when considered at position $\mathbf{r}^{\prime}$. We now choose $u$ to be a harmonic function $(\Delta u=0)$ in $\Omega$, and $v(\mathbf{r})=-G\left(\mathbf{r}-\mathbf{r}^{\prime}\right)$. Using (30) we obtain the third Green identity [6] below, in which $\left(\partial_{\mathbf{n}} u\right)^{-}$and $u^{-}$ denote boundary values taken on the inner side of the boundary

\footnotetext{
${ }^{12}$ Sometimes called the third. The numbering of Green identities varies among authors.
} 


\begin{tabular}{|c|c|c|c|c|c|c|c|c|c|c|c|c|c|c|}
\hline \multirow{12}{*}{ Two-layer head model } & & & \multicolumn{3}{|c|}{ Degrees of freedom } & \multicolumn{3}{|c|}{ Assembly time [s] } & \multicolumn{3}{|c|}{ Factorization time $[\mathrm{s}]$} & \multicolumn{3}{|c|}{ Estimated accuracy (rel.) } \\
\hline & vertices & faces & $1 \mathrm{c}$ & $2 \mathrm{c}$ & 3 & $1 \mathrm{c}$ & $2 \mathrm{c}$ & 3 & $1 \mathrm{c}$ & $2 \mathrm{c}$ & 3 & $1 \mathrm{c}$ & $2 \mathrm{c}$ & 3 \\
\hline & 200 & 196 & 200 & 200 & 396 & 47 & 47 & 10 & $\overline{0}$ & $\overline{0}$ & $\overline{0}$ & 0.152 & 0.153 & 0.132 \\
\hline & 400 & 792 & 400 & 400 & 796 & 201 & 194 & 50 & 0 & 0 & 4 & 0.109 & 0.106 & 0.092 \\
\hline & 600 & 1192 & 600 & 600 & 1196 & 461 & 656 & 155 & 1 & 1 & 10 & 0.090 & 0.088 & 0.078 \\
\hline & 800 & 1592 & 800 & 800 & 1596 & 833 & 951 & 426 & 1 & 2 & $\overline{24}$ & 0.080 & 0.081 & 0.072 \\
\hline & 1000 & 1992 & 1000 & 1000 & 1996 & 2014 & 1968 & 543 & 2 & 2 & 52 & 0.070 & 0.070 & 0.064 \\
\hline & 1200 & 2392 & 1200 & 1200 & 2396 & 2904 & 2839 & 1374 & 2 & 3 & 70 & 0.067 & 0.060 & 0.062 \\
\hline & 1400 & 2792 & 1400 & 1400 & 2796 & 3959 & 3582 & 1807 & 3 & 4 & 92 & 0.062 & 0.065 & 0.059 \\
\hline & 1600 & 3192 & 1600 & 1600 & 3196 & 4045 & 4081 & 3688 & 4 & 5 & 108 & 0.057 & 0.041 & 0.053 \\
\hline & 1800 & 3592 & 1800 & 1800 & 3596 & 8101 & 7915 & 4222 & 5 & 5 & 150 & 0.055 & 0.029 & 0.051 \\
\hline & 2000 & 3992 & 2000 & 3000 & 3996 & 9395 & 10462 & 4828 & 12 & 12 & 333 & 0.051 & N/A & 0.049 \\
\hline
\end{tabular}

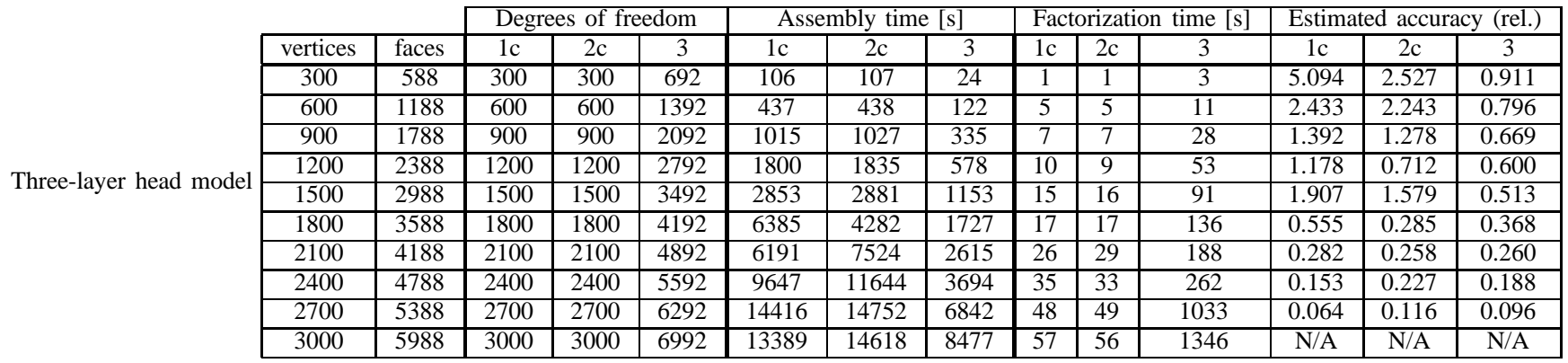

TABLE II

The comparison of the single-layer (1c), double-layer (2c) and symmetric (3) methods applied on a set of progressively fi ner realistically shaped head models with varying number of vertices and faces. The tables show the total number of unknowns, the assembly time for system matrices, the time needed for their (LU) factorization and the estimation of the achieved relative accuracy (see text). In the two-layer model (top) we used the double-layer method as a reference as all the methods converge close to a common solution. In the three-layer model (bottom) we compared each method with its own result on the fi nest mesh because their results, even at the higher resolution of 1000 points per layer, were still too different. N/A (not applicable) indicates that this solution is chosen as the ground-truth.

with respect to the normal field $\mathbf{n}$

$$
\begin{gathered}
\int_{\partial \Omega} G\left(\mathbf{r}-\mathbf{r}^{\prime}\right)\left(\partial_{\mathbf{n}^{\prime}} u\right)^{-}\left(\mathbf{r}^{\prime}\right)-\partial_{\mathbf{n}^{\prime}} G\left(\mathbf{r}-\mathbf{r}^{\prime}\right) u^{-}\left(\mathbf{r}^{\prime}\right) \mathrm{d} s\left(\mathbf{r}^{\prime}\right)= \\
\begin{cases}u(\mathbf{r}) & \text { if } \mathbf{r} \in \Omega \\
u^{-}(\mathbf{r}) / 2 & \text { if } \mathbf{r} \in \partial \Omega \\
0 & \text { otherwise } .\end{cases}
\end{gathered}
$$

This important result shows that a harmonic function $u$ inside a volume $\Omega$ is completely determined by its internal boundary values and those of its normal derivative. To make the notation more compact, we define

$$
\mathscr{P}_{S, \mathbf{n}}^{ \pm}(u)=\int_{S} G\left(\mathbf{r}-\mathbf{r}^{\prime}\right)\left(\partial_{\mathbf{n}^{\prime}} u\right)^{ \pm}\left(\mathbf{r}^{\prime}\right)-\partial_{\mathbf{n}^{\prime}} G\left(\mathbf{r}-\mathbf{r}^{\prime}\right) u^{ \pm}\left(\mathbf{r}^{\prime}\right) \mathrm{d} s\left(\mathbf{r}^{\prime}\right)
$$

and

$$
\chi_{\Omega} u(\mathbf{r})= \begin{cases}u(\mathbf{r}) & \text { if } \mathbf{r} \in \Omega \\ \lim _{\mathbf{r}^{\prime} \rightarrow \mathbf{r}, \mathbf{r}^{\prime} \in \Omega} u\left(\mathbf{r}^{\prime}\right) / 2 & \text { if } \mathbf{r} \in \partial \Omega \\ 0 & \text { if } \mathbf{r} \in \Omega^{c}\end{cases}
$$

Then (31) can be written as

$$
\mathscr{P}_{\partial \Omega, \mathbf{n}}^{-}(u)=\chi_{\Omega} u
$$

Note that if $\mathbf{r} \in \partial \Omega, \lim _{\mathbf{r}^{\prime} \rightarrow \mathbf{r}, \mathbf{r}^{\prime} \in \Omega} u\left(\mathbf{r}^{\prime}\right)=u^{-}(\mathbf{r})$ with respect to a normal field $\mathbf{n}$ on $\partial \Omega$ pointing outside $\Omega$. This is the orientation assumed on the left-hand sides of (31) and (32). Considering a normal vector field $\mathbf{n}_{-}=-\mathbf{n}$ which now points inside the domain $\Omega$, then partial derivatives change signs, and the third Green identity (31) becomes

$$
\begin{array}{r}
-\int_{\partial \Omega} G\left(\mathbf{r}-\mathbf{r}^{\prime}\right)\left(\partial_{\mathbf{n}_{-}^{\prime}} u\right)^{+}\left(\mathbf{r}^{\prime}\right)-\partial_{\mathbf{n}_{-}^{\prime}} G\left(\mathbf{r}-\mathbf{r}^{\prime}\right) u^{+}\left(\mathbf{r}^{\prime}\right) \mathrm{d} s\left(\mathbf{r}^{\prime}\right)= \\
\begin{cases}u(\mathbf{r}) & \text { if } \mathbf{r} \in \Omega \\
u^{+}(\mathbf{r}) / 2 & \text { if } \mathbf{r} \in \partial \Omega \\
0 & \text { otherwise },\end{cases}
\end{array}
$$

the ${ }^{\prime}+{ }^{\prime}$ superscript indicating that the values of $u$ and its normal derivative must this time be considered on the side towards which the normal $\mathbf{n}_{-}$is pointing. Therefore, for an inward-pointing normal field,

$$
-\mathscr{P}_{\partial \Omega, \mathbf{n}_{-}}^{+}(u)=\chi_{\Omega} u \text {. }
$$

Note that $\chi_{\Omega}$ is intrinsic to $\Omega$, in the sense that it is independent of any normal orientation on $\partial \Omega$.

Interestingly, the third Green identity (31) is also valid for a hollow ball topology such as depicted in Fig. 2 as $\Omega=\Omega_{2}$ with a boundary consisting of two non connected parts, $S_{1}$ and $S_{2}$.

The relation (32) supposes that the normal field $\mathbf{n}$ points outside the domain $\Omega$. This is not the case in Figure 2, where the normal field on $S_{1}$ (which we call $\mathbf{n}_{1}$ ) points inwards, whereas the normal field on $S_{2}$ (which we call $\mathbf{n}_{2}$ ) points outwards. Decomposing $\partial \Omega=S_{1} \cup S_{2}$, and using the above considerations on the sign of the normal, one can write

$$
\chi_{\Omega} u=-\mathscr{P}_{S_{1}, \mathbf{n}_{1}}^{+}(u)+\mathscr{P}_{S_{2}, \mathbf{n}_{2}}^{-}(u) .
$$




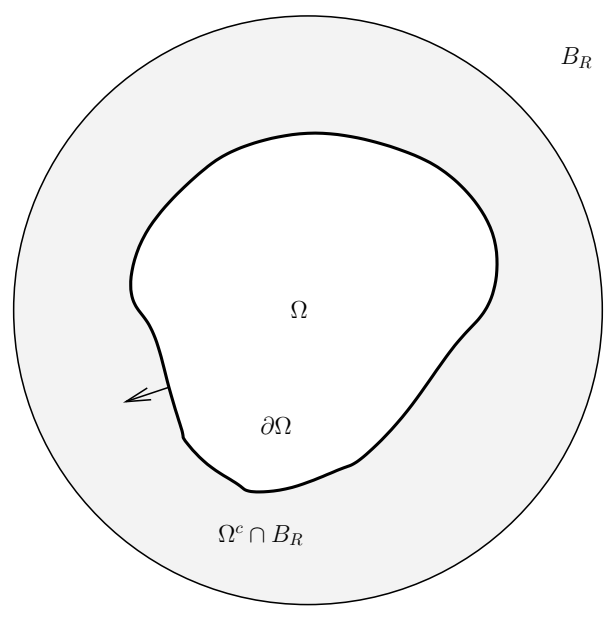

Fig. 9. Two-dimensional slice through a volume $\Omega$ enclosed within a growing ball $B_{R}$, yielding a bounded volume $\Omega^{c} \cap B_{R}$ with a hollow ball topology.

Let us now consider a bounded volume $\Omega$ with the topology of a sphere. We take its connected unbounded complement $\Omega^{c}=\mathbb{R}^{3} \backslash \bar{\Omega}$ and derive the third Green formula for a function $u$ harmonic in $\Omega^{c}$ and satisfying $\mathscr{H}$. To do this, we consider the (bounded) intersection of $\Omega^{c}$ with a ball $B_{R}$ of radius $R$ surrounding $\Omega$, as shown in Figure 9. The volume $\Omega^{c} \cap B_{R}$ has a hollow ball topology and the Green identities hold. As $R$ tends to infinity, the contribution on $\partial B_{R}$ becomes negligible thanks to the fact that both $G$ and $u$ satisfy condition $\mathscr{H}$. This shows that the third Green identity (31) is also valid in an unbounded space $\Omega^{c}$, for harmonic functions $u$ satisfying $\mathscr{H}$. It can be written in the compact form:

$$
\chi_{\Omega^{c}} u=-\mathscr{P}_{\partial \Omega, \mathbf{n}}^{+}(u) .
$$

Combining the third Green identities for $\Omega$ and $\Omega^{c}$ yields the following well-known classical representation theorem, see $[6$, 7] for a complete proof.

Theorem 2 (Representation Theorem for $u$ ) Let $\Omega \subseteq \mathbb{R}^{3}$ be a bounded open set with a regular boundary $\partial \Omega$ and a connected complement space. Let $u:\left(\Omega \cup \Omega^{c}\right) \rightarrow \mathbb{R}$ be a function harmonic $(\Delta u=0)$ in both $\Omega$ and $\Omega^{c}$, satisfying the $\mathscr{H}$ condition, and let further $p\left(\mathbf{r}^{\prime}\right) \stackrel{\text { def }}{=} p_{\mathbf{n}^{\prime}}\left(\mathbf{r}^{\prime}\right)=\partial_{\mathbf{n}^{\prime}} u\left(\mathbf{r}^{\prime}\right)$, where $\mathbf{n}^{\prime}$ is the outward unit normal of $\partial \Omega$ at point $\mathbf{r}^{\prime}$. Then for $\mathbf{r} \notin \partial \Omega$ the following representation holds:

$$
\begin{array}{r}
u(\mathbf{r})=\int_{\partial \Omega}-\partial_{\mathbf{n}^{\prime}} G\left(\mathbf{r}-\mathbf{r}^{\prime}\right)[u]_{\partial \Omega}\left(\mathbf{r}^{\prime}\right)+ \\
G\left(\mathbf{r}-\mathbf{r}^{\prime}\right)[p]_{\partial \Omega}\left(\mathbf{r}^{\prime}\right) \mathrm{d} s\left(\mathbf{r}^{\prime}\right)
\end{array}
$$

and for $\mathbf{r} \in \partial \Omega$

$$
\begin{array}{r}
u^{ \pm}(\mathbf{r})=\mp \frac{[u]_{\partial \Omega}}{2}+\int_{\partial \Omega}-\partial_{\mathbf{n}^{\prime}} G\left(\mathbf{r}-\mathbf{r}^{\prime}\right)[u]_{\partial \Omega}\left(\mathbf{r}^{\prime}\right)+ \\
G\left(\mathbf{r}-\mathbf{r}^{\prime}\right)[p]_{\partial \Omega}\left(\mathbf{r}^{\prime}\right) \mathrm{d} s\left(\mathbf{r}^{\prime}\right) .
\end{array}
$$

The theorem shows that a function $u$ harmonic in $\Omega \cup \Omega^{c}$ and satisfying $\mathscr{H}$ is completely determined by the jumps of $[u]$ and $\left[\partial_{\mathbf{n}} u\right]$ on the interface $\partial \Omega$. Observe that $u$ from (35a) converges to $\left(u^{+}+u^{-}\right) / 2$ on $\partial \Omega$, while the value of $u$ jumps when crossing the boundary. This is shown by the term $\mp \frac{[u]_{\partial \Omega}}{2}$ in (35b).

The single-layer potential (the second part of (35a)) is given explicitly as

$$
u_{s}(\mathbf{r})=\int_{\partial \Omega} G\left(\mathbf{r}-\mathbf{r}^{\prime}\right) \xi\left(\mathbf{r}^{\prime}\right) \mathrm{d} s\left(\mathbf{r}^{\prime}\right)
$$

while the double-layer potential (the first part of (35a)) is written as

$$
u_{d}(\mathbf{r})=\int_{\partial \Omega} \partial_{\mathbf{n}^{\prime}} G\left(\mathbf{r}-\mathbf{r}^{\prime}\right) \mu\left(\mathbf{r}^{\prime}\right) \mathrm{d} s\left(\mathbf{r}^{\prime}\right) .
$$

The function $\xi$ corresponds to a charge density distribution on $\partial \Omega$, while $\mu$ may be viewed as a dipole density. Both potentials (36), (37) satisfy the Laplace equation $\Delta u=0$ in $\Omega \cup \Omega^{c}$ and also satisfy the condition $\mathscr{H}$. Remarkably, with arbitrary functions $\xi, \mu$ from $C^{0}(\partial \Omega)$, both (36) and (37) yield a harmonic function in $C^{2}\left(\Omega \cup \Omega^{c}\right)$.

The single-layer potential is continuous with respect to $\mathbf{r}$, in particular when crossing the boundary $\partial \Omega$. On the other hand its normal derivative is discontinuous when crossing $\partial \Omega$. As proved in [7], the limit values on both sides are

$$
\begin{aligned}
p_{\mathbf{n}}^{ \pm}(\mathbf{r}) & =\partial_{\mathbf{n}} u^{ \pm}(\mathbf{r}) \\
& =\mp \frac{\xi(\mathbf{r})}{2}+\int_{\partial \Omega} \partial_{\mathbf{n}} G\left(\mathbf{r}-\mathbf{r}^{\prime}\right) \xi\left(\mathbf{r}^{\prime}\right) \mathrm{d} s\left(\mathbf{r}^{\prime}\right) \quad \text { for } \mathbf{r} \in \partial \Omega
\end{aligned}
$$

The double-layer potential enjoys the opposite properties. It has a discontinuity when crossing $\partial \Omega$ and the corresponding limit values on both sides of $\partial \Omega$ are

$u^{ \pm}(\mathbf{r})= \pm \frac{\mu(\mathbf{r})}{2}+\int_{\partial \Omega} \partial_{\mathbf{n}^{\prime}} G\left(\mathbf{r}-\mathbf{r}^{\prime}\right) \mu\left(\mathbf{r}^{\prime}\right) \mathrm{d} s\left(\mathbf{r}^{\prime}\right) \quad$ for $\mathbf{r} \in \partial \Omega$

The normal derivative of a double-layer potential is continuous when crossing $\partial \Omega$. Taking the derivative of (37) in the direction $\mathbf{n}$ at $\mathbf{r}$, we write

$$
p(\mathbf{r})=p_{\mathbf{n}}(\mathbf{r})=\partial_{\mathbf{n}} u(\mathbf{r})=\int_{\partial \Omega} \partial_{\mathbf{n}, \mathbf{n}^{\prime}}^{2} G\left(\mathbf{r}-\mathbf{r}^{\prime}\right) \mu\left(\mathbf{r}^{\prime}\right) \mathrm{d} s\left(\mathbf{r}^{\prime}\right) .
$$

A subtle point here is that the kernel $\partial_{\mathbf{n}, \mathbf{n}^{\prime}}^{2} G\left(\mathbf{r}-\mathbf{r}^{\prime}\right)$ is not an integrable function for $\mathbf{r}^{\prime} \rightarrow \mathbf{r}$. We therefore need to treat $p$ as a distribution, defined through scalar products with suitable test functions. This is unlike $[p]$ which is continuous on $\partial \Omega$.

Given these definitions, we can reinterpret (35a) as showing that a function $u$ harmonic in $\Omega \cup \Omega^{c}$ is represented as the sum of a single-layer potential $[6,7]$ corresponding to $\xi=[p]$ and a double-layer potential corresponding to $\mu=-[u]$.

The equations concerning $p$ are summarized in Theorem 3 below, which extends Theorem 2 to the directional and normal derivatives of $u$.

Theorem 3 (Representation Theorem for $p$ ) Let $\Omega \subseteq \mathbb{R}^{3}$ be a bounded open set with a regular boundary $\partial \Omega$. Let $u:(\Omega \cup$ $\left.\Omega^{c}\right) \rightarrow \mathbb{R}$ be a function harmonic $(\Delta u=0)$ in both $\Omega$ and $\Omega^{c}$, 
satisfying condition $\mathscr{H}$. Let also $\mathbf{n}\left(\right.$ resp. $\mathbf{n}^{\prime}$ ) be the unit normal to $\partial \Omega$ at point $\mathbf{r}$ (resp. $\mathbf{r}^{\prime}$ ) and $\mathbf{m}$ an arbitrary unit vector at $\mathbf{r}$. Then for $\mathbf{r} \notin \partial \Omega$ the following representation holds:

$$
\begin{array}{r}
p_{\mathbf{m}}(\mathbf{r})=\int_{\partial \Omega}-\partial_{\mathbf{m}, \mathbf{n}^{\prime}}^{2} G\left(\mathbf{r}-\mathbf{r}^{\prime}\right)[u]_{\partial \Omega}\left(\mathbf{r}^{\prime}\right)+ \\
\partial_{\mathbf{m}} G\left(\mathbf{r}-\mathbf{r}^{\prime}\right)[p]_{\partial \Omega}\left(\mathbf{r}^{\prime}\right) \mathrm{d} s\left(\mathbf{r}^{\prime}\right)
\end{array}
$$

and for $\mathbf{r} \in \partial \Omega$

$$
\begin{array}{r}
p^{ \pm}(\mathbf{r}) \stackrel{\text { def }}{=} p_{\mathbf{n}}^{ \pm}(\mathbf{r})=\mp \frac{[p]_{\partial \Omega}}{2}+\int_{\partial \Omega}-\partial_{\mathbf{n}, \mathbf{n}^{\prime}}^{2} G\left(\mathbf{r}-\mathbf{r}^{\prime}\right)[u]_{\partial \Omega}\left(\mathbf{r}^{\prime}\right)+ \\
\partial_{\mathbf{n}} G\left(\mathbf{r}-\mathbf{r}^{\prime}\right)[p]_{\partial \Omega}\left(\mathbf{r}^{\prime}\right) \mathrm{d} s\left(\mathbf{r}^{\prime}\right) .
\end{array}
$$

Analogously to $u$, the derivative $p=p_{\mathbf{n}}$ given by (40a) for $\mathbf{m}=\mathbf{n}$ converges to $p=\left(p^{+}+p^{-}\right) / 2$ on $\partial \Omega$ in the distributional sense.

\section{B. Multiple interfaces}

Having in view the layered model depicted in Fig. 1, we extend Theorem 2 to a nested domain topology. As we have seen in Section III-G, it suffices to consider the case of three nested domains as in Fig. 2.

Proposition 1 Let $\Omega_{1}, \Omega_{2}, \Omega_{3}$ be disjoint open sets such that $\bar{\Omega}_{1} \cup \bar{\Omega}_{2} \cup \Omega_{3}=\mathbb{R}^{3}$, separated by regular boundaries $\partial \Omega_{1} \cap$ $\partial \Omega_{2}=S_{1}, \partial \Omega_{2} \cap \partial \Omega_{3}=S_{2}$, and $\partial \Omega_{1} \cap \partial \Omega_{3}=\emptyset$. Let $u$ : $\left(\Omega_{1} \cup \Omega_{2} \cup \Omega_{3}\right) \rightarrow \mathbb{R}$ be harmonic and satisfy $\mathscr{H}$. Then (35a) and (35b) hold with $\partial \Omega=S_{1} \cup S_{2}$.

Proof: To prove this proposition, we use the third Green identities (32) in $\Omega_{1}$ and (34) in $\Omega_{3}$, and the variant (33) in $\Omega_{2}$. We assume that the normal vector fields $\mathbf{n}_{1}$ on $S_{1}$ and $\mathbf{n}_{2}$ on $S_{2}$ point globally outward (as in Fig. 2). We have

$$
\begin{aligned}
& \chi_{\Omega_{1}} u=\mathscr{P}_{S_{1}, \mathbf{n}_{1}}^{-}(u), \\
& \chi_{\Omega_{2}} u=-\mathscr{P}_{S_{1}, \mathbf{n}_{1}}^{+}(u)+\mathscr{P}_{S_{2}, \mathbf{n}_{2}}^{-}(u), \\
& \chi_{\Omega_{3}} u=-\mathscr{P}_{S_{2}, \mathbf{n}_{2}}^{+}(u) .
\end{aligned}
$$

Summing up the three contributions gives

$$
\begin{aligned}
\chi_{\Omega_{1}} u+\chi_{\Omega_{2}} u+\chi_{\Omega_{3}} u= & \left(\mathscr{P}_{S_{1}, \mathbf{n}_{1}}^{-}(u)-\mathscr{P}_{S_{1}, \mathbf{n}_{1}}^{+}(u)\right)+ \\
& \left(\mathscr{P}_{S_{2}, \mathbf{n}_{2}}^{-}(u)-\mathscr{P}_{S_{2}, \mathbf{n}_{2}}^{+}(u)\right) .
\end{aligned}
$$

For $\mathbf{r} \in \Omega_{1} \cup \Omega_{2} \cup \Omega_{3}, \chi_{\Omega_{1}} u(\mathbf{r})+\chi_{\Omega_{2}} u(\mathbf{r})+\chi_{\Omega_{3}} u(\mathbf{r})=u(\mathbf{r})$, and we obtain (35a). For $\mathbf{r} \in S_{1} \cup S_{2}, \chi_{\Omega_{1}} u(\mathbf{r})+\chi_{\Omega_{2}} u(\mathbf{r})+$ $\chi_{\Omega_{3}} u(\mathbf{r})=\frac{u^{+}(\mathbf{r})+u^{-}(\mathbf{r})}{2}$. Since $u^{+}=-\frac{1}{2}[u]_{\partial \Omega}+$ $\frac{u^{+}+u^{-}}{2}$ and $u^{-}=\frac{1}{2}[u]_{\partial \Omega}+\frac{u^{+}+u^{-}}{2}$, we obtain (35b).

Theorem 3 also holds in the case of a nested volume topology, but we do not provide the detailed proof here.

The single and double potentials can also easily be applied in the case of our layered model from Fig.1. For the singlelayer potential, we write:

$$
u_{s}(\mathbf{r})=\sum_{i=1}^{N} \int_{S_{i}} G\left(\mathbf{r}-\mathbf{r}^{\prime}\right) \xi_{S_{i}}\left(\mathbf{r}^{\prime}\right) \mathrm{d} s\left(\mathbf{r}^{\prime}\right)
$$

where each $\xi_{S_{i}}$ is defined on the corresponding surface $S_{i}$. The properties from the single interface case are trivially satisfied thanks to additivity, namely $\left[u_{s}\right]_{i}=0$ and $\left[\partial_{\mathbf{n}} u_{s}\right]_{i}=\xi_{i}$. More specifically,

$$
\begin{aligned}
p_{\mathbf{n}}^{ \pm}(\mathbf{r}) & =\partial_{\mathbf{n}} u_{s}^{ \pm}(\mathbf{r}) \\
& =\mp \frac{\xi_{S_{j}}(\mathbf{r})}{2}+\sum_{i=1}^{N} \int_{S_{i}} \partial_{\mathbf{n}} G\left(\mathbf{r}-\mathbf{r}^{\prime}\right) \xi_{S_{i}}\left(\mathbf{r}^{\prime}\right) \mathrm{d} s\left(\mathbf{r}^{\prime}\right),
\end{aligned}
$$

for $\mathbf{r} \in S_{j}$. Similarly, for the double-layer potential, we have

$$
u_{d}(\mathbf{r})=\sum_{i=1}^{N-1} \int_{S_{i}} \partial_{\mathbf{n}^{\prime}} G\left(\mathbf{r}-\mathbf{r}^{\prime}\right) \mu_{S_{i}}\left(\mathbf{r}^{\prime}\right) \mathrm{d} s\left(\mathbf{r}^{\prime}\right),
$$

with $\left[u_{d}\right]_{i}=-\mu_{S_{i}},\left[\partial_{\mathbf{n}} u_{d}\right]=0$ and

$$
u_{d}^{ \pm}(\mathbf{r})= \pm \frac{\mu_{S_{j}}(\mathbf{r})}{2}+\sum_{i=1}^{N} \int_{S_{i}} \partial_{\mathbf{n}^{\prime}} G\left(\mathbf{r}-\mathbf{r}^{\prime}\right) \mu_{S_{i}}\left(\mathbf{r}^{\prime}\right) \mathrm{d} s\left(\mathbf{r}^{\prime}\right),
$$

for $\mathbf{r} \in S_{j}$.

\section{Uniqueness}

The integral representation in terms of $\mu=-[u]$ and $\xi=[p]$ is unique, if $u$ and $p$ are considered as a pair [7]. In other words, there is only one pair of $(\mu, \xi)$ generating a given set of pairs $\left(u^{-}, p^{-}\right),\left(u^{+}, p^{+}\right)$, or $([u],[p])$. However, this is no longer true if only $p^{-}$is given (interior Neumann problem), as any constant function can be added to $u$. Physically, this means that the potential is only known up to a constant. To get rid of this indetermination, we can for example choose to impose $\langle u, 1\rangle=0$; other options are possible [33]. In the same spirit, note that for harmonic $u$, Stokes' theorem necessarily imposes $\langle p, 1\rangle=0$ on any closed surface.

\section{Weak regularity}

We need to extend Theorems 2 and 3 to non-regular surfaces $^{13}$, such as for example a triangulated surface which is not regular on the edges and at the vertices. We find that the equations in Theorems 2 and 3 hold, provided that there are not "too many" singular points (their set is of zero measure) and that we only evaluate the integrals in (35b), (40b) at the regular points. If needed, the values/limits on the surface can still be calculated even at the singular points by a more complex expression involving inner and outer spherical angles $[10,43]$. We will avoid this complication here by concentrating on the Galerkin method that gives better results and does not require pointwise values.

In a similar vein, we can relax the continuity requirements on $\mu=-[u], \xi=[p]$, in order to approximate them by some $\tilde{\mu}$, $\tilde{\xi}$. Only piecewise continuity is necessary for the convergence of the integrals in Theorems 2 and 3 (again with a zero measure set of discontinuity points) if we do not evaluate (35b) and $(40 \mathrm{~b})$ at the points of discontinuity.

\footnotetext{
${ }^{13} \mathrm{~A}$ surface is regular if it can be locally approximated by a linear function everywhere.
} 


\section{REFERENCES}

[1] J. Phillips, R. Leahy, J. Mosher, and B. Timsari, 'Imaging neural activity using meg and eeg," IEEE Engineering in Medicine and Biology Magazine, vol. 16, no. 3, pp. 34-42, 1997.

[2] J. Sarvas, "Basic mathematical and electromagnetic concepts of the biomagnetic inverse problem," Phys. Med. Biol., vol. 32, no. 1, pp. 1122, 1987.

[3] M. Häamaläainen, R. Hari, R. J. IImoniemi, J. Knuutila, and O. V. Lounasmaa, "Magnetoencephalography - theory, instrumentation, and applications to noninvasive studies of the working human brain," Reviews of Modern Physics, vol. 65, no. 2, pp. 413-497, Apr. 1993.

[4] D. B. Geselowitz, "On the magnetic fi eld generated outside an inhomogeneous volume conductor by internal volume currents," IEEE Trans. Magn., vol. 6, pp. 346-347, 1970.

[5] O. Faugeras, F. Clément, R. Deriche, R. Keriven, T. Papadopoulo, J. Roberts, T. Viéville, F. Devernay, J. Gomes, G. Hermosillo, P. Kornprobst, and D. Lingrand, "The inverse EEG and MEG problems: The adjoint space approach I: The continuous case," INRIA, Tech. Rep. 3673, May 1999. [Online]. Available: http: //www.inria.fr/RRRT/RR-3673.html

[6] M. Bonnet, Equations intégrales et éléments de frontière. CNRS Editions, Eyrolles, 1995.

[7] J.-C. Nédélec, Acoustic and Electromagnetic Equations. Springer Verlag, 2001.

[8] J. Rahola and S. Tissari, "Iterative solution of dense linear systems arising from the electrostatic integral equation," Phys. Med. Biol., no. 47, pp. 961-975, 2002.

[9] — , 'Iterative solution of dense linear systems arising from boundary element formulations of the biomagnetic inverse problem," CERFACS, Tech. Rep. TR/PA/98/40, 1998, toulouse, France.

[10] A. S. Ferguson and G. Stroink, "Factors affecting the accuracy of the boundary element method in the forward problem - I: Calculating surface potentials," IEEE Trans. Biomed. Eng., vol. 44, no. 11, pp. 11391155, Nov. 1997.

[11] M. Clerc, R. Keriven, O. Faugeras, J. Kybic, and T. Papadopoulo, "The fast multipole method for the direct E/MEG problem," in Proceedings of ISBI. Washington, D.C.: IEEE, NIH, July 2002. [Online]. Available: http://www.biomedicalimaging.org/

[12] S. Tissari and J. Rahola, 'Error analysis of a new Galerkin method to solve the forward problem in MEG and EEG using the boundary element method," CERFACS, Tech. Rep. TR/PA/98/39, 1998, toulouse, France.

[13] M. S. H"am"al"ainen and J. Sarvas, "Realistic conductivity geometry model of the human head for interpretation of neuromagnetic data," IEEE Trans. Biomed. Eng., vol. 36, no. 2, pp. 165-171, Feb. 1989.

[14] S. Tissari and J. Rahola, "A precorrected-FFT method to accelerate the solution of the forward problem in MEG," in Proceedings of BIOMAG, 2002. [Online]. Available: http://biomag2002.uni-jena.de/

[15] Z. Zhang and D. L. Jewett, 'Insidious errors in dipole localization parameters at a single time-point due to model misspecifi cation of number of shells," Electroencephalography and clinical Neurophysiology, no. 88, pp. 1-11, 1993.

[16] R. Leahy, J. Mosher, M. Spencer, M. Huang, and J. Lewine, "A study of dipole localization accuracy for MEG and EEG using a human skull phantom,' Los Alamos National Laboratory, Los Alamos Technical Report LA-UR-98-1442, Mar. 1998, revision of LA-UR-97-4804.

[17] D. B. Geselowitz, 'On bioelectric potentials in an homogeneous volume conductor," Biophysics Journal, vol. 7, pp. 1-11, 1967.

[18] F. Babiloni, C. Del Gratta, F. Carducci, C. Babiloni, G. M. Roberti, V. Pizzella, P. M. Rossini, G. L. Romani, and A. Urbano, 'Combined high resolution EEG and MEG data for linear inverse estimate of human event-related cortical activity," in Proceedings of the 20th annual int. conf. of the IEEE Eng. Med. Biol. Society, no. 4, 1998, pp. 2151-2154.

[19] J. C. de Munck, "A linear discretization of the volume conductor boundary integral equation using analytically integrated elements," IEEE Trans. Biomed. Eng., vol. 39, no. 9, pp. 986-990, Sept. 1992.

[20] J. C. Mosher, R. B. Leahy, and P. S. Lewis, "EEG and MEG: Forward solutions for inverse methods," IEEE Transactions on Biomedical Engineering, vol. 46, no. 3, pp. 245-259, Mar. 1999.

[21] J. C. Mosher, R. M. Leahy, and P. S. Lewis, 'Matrix kernels for the forward problem in EEG and MEG," Los Alamos, Tech. Rep. LA-UR97-3812, 1997.

[22] N. G. Gencer and I. O. Tanzer, 'Forward problem solution of electromagnetic source imaging using a new BEM formulation with high-order elements," Phys. Med. Biol., vol. 44, no. 9, pp. 2275-2287, 1999.
[23] L. J. Gray and G. H. Paulino, 'Symmetric Galerkin boundary integral formulation for interface and multi-zone problems," Internat. J. Numer. Methods Eng., vol. 40, no. 16, pp. 3085-3103, 1997.

[24] J. B. Layton, S. Ganguly, C. Balakrishna, and J. H. Kane, "A symmetric Galerkin multi-zone boundary element formulation," Internat. J. Numer. Methods Eng., vol. 40, no. 16, pp. 2913-2931, 1997.

[25] S. H. Christiansen, "Résolution des équations intégrales pour la diffraction d'ondes acoustiques et électromagnétiques," Ph.D. dissertation, Ecole Polytechnique, 2002.

[26] R. Dautray and J.-L. Lions, Mathematical Analysis and Numerical Methods for Science and Technology. Springer, 2000, 6 volumes.

[27] J. L"otj"onen, "Construction of boundary element models in bioelectromagnetism," Ph.D. dissertation, Helsinki University of Technology, Espoo, Finland, 2000.

[28] D. Van 't Ent, J. C. De Munck, and A. L. Kaas, "A fast method to derive realistic BEM models for E/MEG source reconstruction," IEEE Trans. Biomed. Eng., vol. 48, no. 12, Dec. 2001.

[29] D. R. Wilton, S. M. Rao, A. W. Glisson, D. H. Schaubert, O. M. AlBundak, and C. M. Butler, 'Potential integrals for uniform and linear source distributions on polygonal and polyhedral domains," IEEE Trans. Antenn. Propag., vol. 32, no. 3, pp. 276-281, Mar. 1984.

[30] A. S. Ferguson, X. Zhang, and G. Stroink, "A complete linear discretization for calculating the magnetic fi eld using the boundary element method," IEEE Trans. Biomed. Eng., vol. 41, no. 5, pp. 455-459, May 1994.

[31] J. N. Lyness and D. Jespersen, 'Moderate degree symmetric quadrature rules for the triangle," J. Inst. Maths Applics, vol. 15, pp. 19-32, 1975.

[32] T. F. Chan, "Defated decomposition solution of nearly singular systems," SIAM J. Numer. Anal., vol. 21, pp. 739-754, 1984.

[33] G. Fischer, B. Tilg, R. Modre, F. Hanser, B. Messnarz, and P. Wach, 'On modeling the Wilson terminal in the Boundary and Finite Element Method," IEEE Trans. Biomed. Eng., vol. 49, no. 3, pp. 217-224, Mar. 2002.

[34] G. Strang and G. Fix, "A Fourier analysis of the fi nite element variational method," in Constructive Aspect of Functional Analysis. Rome, Italy: Cremonese, 1971, pp. 796-830.

[35] R. Barret, M. Berry, T. F. Chan, J. Demmel, J. Donato, J. Dongarra, V. Eijkhout, R. Pozo, C. Romine, and H. van der Vonst, Templates for the Solution of Linear Systems: Building Blocks for Iterative Methods. Philadelphia: SIAM, 1994, available from netlib.

[36] J. R. Phillips and J. K. White, "A precorrected-FFT method for electrostatic analysis of complicated 3-D structures," IEEE Trans. CAD Int. Circ. Syst., vol. 16, no. 10, Oct. 1997.

[37] J. C. De Munck, "The potential distribution in a layered anisotropic spheroidal volume conductor," J. Appl. Phys, vol. 2, no. 64, pp. 464470, July 1988 .

[38] Z. Zhang, "A fast method to compute surface potentials generated by dipoles within multilayer anisotropic spheres," Phys. Med. Biol., vol. 40, pp. 335-349, 1995.

[39] U. Schmitt, A. K. Louis, F. Darvas, H. Buchner, and M. Fuchs, "Numerical aspects of spatio-temporal current density reconstruction from EEG/MEG-data," IEEE Trans. Med. Imag., vol. 20, no. 4, pp. 314-324, 2001.

[40] R. Van Uitert and C. Johnson, 'Can a spherical model substitute for a realistic head model in forward and inverse MEG simulations?" in Proceedings of BIOMAG, 2002. [Online]. Available: http://biomag2002. uni-jena.de/

[41] J. Kybic and M. Clerc, 'Symmetric bem and multiscale fast multipole method for the e/meg problem," in 4th International Symposium on Noninvasive Functional Source Imaging within the human brain and heart, Chieti, Sept. 2003.

[42] L. Evans, Partial Differential Equations, ser. Graduate Studies in Mathematics. Proceedings of the American Mathematical Society, 1998, vol. 19.

[43] J. W. H. Meijs, O. W. Weier, M. J. Peters, and A. van Oosterom, 'On the numerical accuracy of the boundary element method," IEEE Trans. Biomed. Eng., vol. 36, pp. 1038-1049, 1989. 